\title{
Unilateral facial injection of Botulinum neurotoxin $A$ attenuates bilateral trigeminal neuropathic pain and anxiety-like behaviors through inhibition of TLR2-mediated neuroinflammation in mice
}

Wei-Jia Chen ${ }^{1 \dagger}$, Jing-Qi Niu ${ }^{1,2+}$, Yi-Ting Chen ${ }^{3+}$, Wen-Jing Deng ${ }^{1}$, Ying-Ying $X u^{1}$, Jing Liu ${ }^{1}$, Wei-Feng Luo ${ }^{1,4^{*}}$ and Tong $\operatorname{Liu}^{5,6,7^{*}}$

\begin{abstract}
Objectives: In this study, we investigated the possible analgesic effects of Botulinum toxin type A (BoNT/A) on trigeminal neuralgia (TN). A modified TN mouse model was established by chronic constriction injury of the distal infraorbital nerve (dloN-CCl) in mice, and the possible roles of microglia toll-like receptor 2 (TLR2) and neuroinflammation was investigated.

Methods: Male C57BL/6 mice were divided into 3 groups, including sham group, vehicle-treated TN group and BoNT/Atreated TN group. Bilateral mechanical pain hypersensitivity, anxiety-like and depressive-like behaviors were evaluated by using von Frey test, open field, elevated plus-maze testing, and forced swimming test in mice, respectively. The mRNA or protein expression levels of toll-like receptors (TLRs), glia activation markers and proinflammatory factors in the trigeminal nucleus caudalis (TNC) were tested by RT-qPCR, immunofluorescence and Western blotting. We also tested the pain behaviors of $\mathrm{TN}$ in $\mathrm{TI}^{-/-}$mice.

Results: We found that unilateral subcutaneous injection of BoNT/A into the whisker pad on the ipsilateral side of dloN$\mathrm{CCl}$ mice significantly attenuated bilateral mechanical pain hypersensitivity and anxiety-like behaviors induced by dloN$\mathrm{CCl}$ surgery in mice. The dloN-CCl surgery significantly up-regulated the expression of TLR2, MyD88, CD11b (a microglia marker), IL-1 $\beta$, TNF- $a$ and IL-6 in the ipsilateral TNC in mice, and BoNT/A injection significantly inhibited the expression of these factors. Immunostaining results confirmed that BoNT/A injection significantly inhibited the microglia activation in the ipsilateral TNC in dloN-CCI mice. TLR2 deficiency also alleviated bilateral mechanical pain hypersensitivity and the upregulation of MyD88 expression in the TNC of dloN-CCl mice.
\end{abstract}

\footnotetext{
*Correspondence: Iwfwxx@126.com; tongliu@ntu.edu.cn

${ }^{+}$Wei-Jia Chen, Jing-Qi Niu and Yi-Ting Chen contributed equally to this work.

'Department of Neurology, The Second Affiliated Hospital of Soochow

University, 1055 Sanxiang Road, Suzhou 215004, China

${ }^{5}$ Institute of Pain Medicine and Special Environmental Medicine, Nantong

University, Nantong 226019, China

Full list of author information is available at the end of the article
}

C C The Author(s). 2021 Open Access This article is licensed under a Creative Commons Attribution 4.0 International License, which permits use, sharing, adaptation, distribution and reproduction in any medium or format, as long as you give appropriate credit to the original author(s) and the source, provide a link to the Creative Commons licence, and indicate if changes were made. The images or other third party material in this article are included in the article's Creative Commons licence, unless indicated otherwise in a credit line to the material. If material is not included in the article's Creative Commons licence and your intended use is not permitted by statutory regulation or exceeds the permitted use, you will need to obtain permission directly from the copyright holder. To view a copy of this licence, visit http://creativecommons.org/licenses/by/4.0/ The Creative Commons Public Domain Dedication waiver (http://creativecommons.org/publicdomain/zero/1.0/) applies to the data made available in this article, unless otherwise stated in a credit line to the data. 
Conclusion: These results indicate that unilateral injection of BoNT/A attenuated bilateral mechanical pain hypersensitivity and anxiety-like behaviors in dloN-CCl mice, and the analgesic effects of BoNT/A may be associated with the inhibition of TLR2-mediated neuroinflammation in the TNC.

Keywords: Trigeminal neuralgia, Anxiety, Microglia, Innate immunity, Toll-like receptor 2 (TLR2), Botulinum toxin type A

\section{Background}

Trigeminal neuralgia (TN) is possible one of the most severe pain suffered by human [1]. The onset of the disease is usually between 50 and 70 years old. Clinical manifestation of TN often presents as a severe pain like a cut, stab, or electric shock [1]. Current treatments for TN include pharmacological and surgical treatments. Of note, most TN patients are resistant to opioids (such as morphine), which brings a big challenge for the management of TN. Clinically, antiepileptic drugs are often used medications, include carbamazepine, oxcarbazepine, and gabapentin [2]. Along with the TN disease progression, TN patients require higher doses of analgesics to control pain, which leads to more unwanted side effects [2]. For the case of refractory TN, surgical procedures may be a considered strategy, including microvascular decompression and gamma knife surgery [3]. However, abovementioned surgical procedures may have some risks, including ataxia, brainstem injury, and cranial nerve palsy [3]. Therefore, TN patients are in urgent need of a new treatment without side effects.

Under chronic pain conditions, bilateral mechanical pain hypersensitivity in response to unilateral injury is also well-known as mirror image pain [4]. The mechanisms underlying mirror image pain are largely unclear yet. As previously reported, nerve damage increased activity in the contralateral S1 cortex associated with chronic nociception [4]. Previous work demonstrated that early administration of agents that inhibit satellite glial activation or reduce its production of nerve growth factor (NGF) can be effective in relieving mirror image pain induce by spinal nerve ligation $(\mathrm{SNL})$ in rats $[4,5]$. However, the detailed mechanisms underlying mirror image pain are still elusive and effective treatments are not available clinically yet.

Botulinum toxins are exotoxins produced by the Clostridium botulinum, a gram-positive anaerobic bacillus, during the reproduction process [6]. Botulinum toxin type A (BoNT/A) produces muscle relaxation by inhibiting the release of acetylcholine at the neuromuscular junction, which prevents the downward neurotransmission of nerve impulses and achieves the effect of denervation, resulting in muscle relaxation [6]. Nowadays, BoNT/A is the widely used in clinical practice. In 2002, Micheli reported the first case in which BoNT/A was used to treat $\mathrm{TN}$ with significant therapeutic benefits [7]. Our previous study demonstrated that BoNT/A injection attenuates depression-like behavior induced by chronic stress in mice, possible via upregulation of the expression of BDNF in the hippocampus [8]. Depression and anxiety are often observed in patients with chronic pain, with a prevalence of $30 \%$ to $50 \%$ [9]. Recent studies have shown that neuropathic pain causes anxiety and depression-like behaviour in mice [9]. However, the mechanisms underlying analgesic, anti-depressant or anxiolytic of BoNT/A therapy is still largely unclear.

Injury-induced synaptic plasticity in the pain circuits of the central nervous system (including spinal cord and brain), also known as central sensitization, is responsible for the maintenance of persistent pain and/or widespread pain beyond the initial site of injury of the body [10]. Glia (such as microglia and astrocyte) activation has been demonstrated to be critical for the development of central sensitization, which contributes to the pathogenesis of neuropathic pain [10]. A key aspect of microglia responding to nerve injury is the proliferation (microgliosis) and activation of microglia in the central nervous system (CNS), including the spinal cord. Microglia activation caused by peripheral nerve injury is accompanied by the development of pain hypersensitivity and blocking microglia activation can reduce pain behaviors in animal models. Activated microglia can release pro-inflammatory mediators, such as tumor necrosis factor- $\alpha$ (TNF- $\alpha$ ), interleukin-1 $\beta$ (IL-1 $\beta$ ), IL6 , brain-derived neurotrophic factor (BDNF) and prostaglandin E2 (PGE2), which promote the initiation and maintenance of neuropathic pain. However, the inhibitory effect of BoNT/A on microglia activation after nerve injury and the detailed mechanisms has not been investigated [11].

Toll-like receptors (TLRs) are normally expressed in immune and glial cells to regulate innate and adaptive immunity $[12,13]$. TLR2 gene expression is known to be used as a reliable marker of activated microglia in vivo, but its detailed role in microgliosis remains unknown [14]. Previous studies have also suggested that TLR2 is involved in the pathogenesis of neuropathic pain models. However, the role of TLR2 in TN remains unclear. In 2015, Yun Jeong Kim et al. suggested that in macrophages, BoNT/A may act on TLR2 to cause a decrease in mitogen-activated protein kinase (MAPK) signaling pathway, which caused a decrease in the expression of several pro-inflammatory mediators [15]. Therefore, the purpose of this study was to test whether peripheral 
(facial) injection of BoNT/A could relieve $\mathrm{TN}$ and to further explore possible molecular mechanisms. We used chronic constriction injury (CCI) of the distal infraorbital nerve (dIoN) to establish a modified TN model according to previous report [16]. Then, we explored the analgesic role of BoNT/A in TN and its mechanism of action in TNC by using behavioral and molecular biological approaches. Hopefully, we expect to provide an effective BoNT/A treatment for the management of TN.

\section{Methods}

\section{Animals}

Six to eight weeks old male C57BL/6 J mice (weighing over $20 \mathrm{~g}$ ) were provided by experimental animal center of Soochow University. We randomly assigned male C57BL/6 J mice of matched age to the sham-operated group, dIoN-CCI + vehicle group and dIoN-CCI + BoNT/A treated group. The group of mice during testing was blinded to the experimenter of the behavioral test. Male TLR2 knockout mice (Tlr2 ${ }^{-/-}$; stock\#005846) were purchased from the Jackson Laboratory and raised at Soochow University Laboratory Animal Center. They were housed at five to six per cage, habituated to the colony room for a week before the experiments were performed, and kept under a $12 \mathrm{~h}$ light $/ 12 \mathrm{~h}$ night cycle. The rearing environment was maintained at a constant room temperature of $22^{\circ} \mathrm{C}$ and $60 \%-80 \%$ humidity. In addition, water and food were freely available. All procedures were approved by the Animal Care and Use Committee of Soochow University. The number of mice used in each experiments are shown in Table 1. This animal study followed the ARRIVE guidelines [17].

\section{Mouse model of TN}

The chronic constriction injury (CCI) of the distal infraorbital nerve ( $\mathrm{dIoN}$ ) was established following the previous research by Weihua Ding et al. [16]. Without damaging the whiskers, gently shave the facial surface between the mouse's eyes and the whisker pad. Made a $0.5 \mathrm{~cm}$ incision parallel to the midline, starting from the caudal end of the third row of whisker lines and then toward the ipsilateral orbit. The superficial fascia was bluntly separated and the main trunk of the infraorbital nerve (IoN) outside the orbital cavity was exposed in its

Table 1 Animal numbers in each group

\begin{tabular}{llllll}
\hline Experimental group & Behavioral tests & RT-qPCR & WB & IF & Total \\
\hline Sham & $12^{\text {a }}$ & 4 & 4 & 4 & 12 \\
dloN-CCl & $18^{\text {a }}$ & 4 & 10 & 4 & 18 \\
dloN-CCl+BoNT/A & $12^{\text {a }}$ & 4 & 4 & 4 & 12 \\
Tlr ${ }^{-/-}$dloN-CCl & $6^{\text {a }}$ & - & 6 & - & 6 \\
Total & & & & & 48 \\
\hline
\end{tabular}

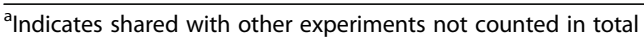

distal segment. Two chromic catgut ligatures (6-0) were tied loosely to the distal portion of the IoN ( $1 \mathrm{~mm}$ distance). To ensure proper constriction of the IoN, the ligatures were applied in such a way that the diameter of the IoN was reduced and superficial vascular circulation was retarded but not severed. Skin incisions were closed with polyester sutures (6-0). The sham-operated group of mice underwent the same surgical procedure, including skin incision and IoN nerve exposure, except that the actual nerve was ligated.

\section{Drugs and administration}

The following reagents were obtained from the sources indicated: BoNT/A (GMP nos S10970037) was obtained from the Lanzhou Institute of Biological Products, Lanzhou, China. Each vial contains $100 \mathrm{U}$ of purified C. Botulinum type A (BoNT/A) neurotoxin complex. The toxin was stored at $-80{ }^{\circ} \mathrm{C}$ in $0.9 \% \mathrm{NaCl}$. All other chemicals used in the study were of analytical grade and obtained from commercial sources. Two weeks after dIoN-CCI surgery, $0.18 \mathrm{U}$ BoNT/A was injected subcutaneously (s.c.) into the whisker pad on the ipsilateral side of the dIoN-CCI mice.

\section{Mechanical pain test}

Von Frey filaments were used to measure mechanical pain in the bilateral whisker pad of mice. We performed pilot experiments to measure mechanical pain by the "up-down method" and found that mice had an obvious positive pain response at $0.008 \mathrm{~g}$. The intensity of the stimulus filaments was set at $0.008 \mathrm{~g}$ and $0.02 \mathrm{~g}$. Mice with positive pain responses were tested as described by Weihua Ding et al. [16]. Each mouse was placed in a small transparent plastic cage for $1 \mathrm{~h}$ to acclimatize to the experimental environment, and the experimenter was blinded to the group assignment of the mice. The number of positive reactions produced by both sides of the mice was counted. Each stimulus was given 10 times on each side and repeated three times. The final mouse mechanical pain was calculated as a percentage of positive responses in the total number of stimuli. We tested the basal mechanical pain positive response rate followed by dIoN-CCI 1 week later and weekly thereafter. To study the analgesic effect of BoNT/A, after BoNT/A injection, mechanical pain testing was performed at $1 \mathrm{~h}, 1$ day, after that, the test was conducted every 4 days.

\section{Open field test}

To evaluate the behavior of three groups of mice in the open field, mice were individually placed into a $40 * 40$ $\mathrm{cm}$ center area of a brightly illuminated open field arena. The bottom was divided into a $4 * 4$ grid, 16 equal sized $\left(10 \mathrm{~cm}^{*} 10 \mathrm{~cm}\right)$ squares. Mice were allowed to explore 
the stage undisturbed for $10 \mathrm{~min}$. Video tracking software was used to count the time the mice entered the central bright area (the middle four squares). And the excretion rate of the three groups of mice during the test was counted.

\section{Elevated plus-maze testing}

Mice were taken individually to the chamber $1 \mathrm{~h}$ before the start of the trial. The elevated plus maze device was made of white opaque plastic plates and an iron trestle table. It consisted of two enclosed arms and two open arms $\left(36 \mathrm{~cm}^{*} 6 \mathrm{~cm}\right)$ fixed to the central platform. There was a $6 \mathrm{~cm}^{*} 6 \mathrm{~cm}$ open section in the center of the maze. The two enclosed arms were surrounded on three sides by $15 \mathrm{~cm}$ high walls, and the two open arms had no walls. The maze was elevated to a height of $60 \mathrm{~cm}$ [18]. When tested, the mice were placed individually in the center of the maze with their heads facing an open arm (the same for all mice). Before the next mouse was tested, the instrument was wiped clean with an absorbent cotton wool. Each mouse was videotaped for $5 \mathrm{~min}$. During the $5 \mathrm{~min}$ test period, the duration and number of entries into the open and closed arms were counted separately (mice with all four limbs entered were included in the count). These measures enabled the calculation of the duration and number of times the mice entered the open or the closed arms. The number of times mice entered the open arms and the duration in the open arms were calculated as a percentage of the total number of entries (sum of open and closed arms entries) and total time (sum of duration in both arms). The anxiolytic effect was represented by an increase in the percentage of the number of times the mouse entered the open arms and the percentage of the time spent in the open arms, but the total number of entries and the total time spent in the arms remained unchanged. On the contrary, the anxiogenic effect was reflected that both percentages decreased without affecting the total number of entries and the duration in both arms.

\section{Forced swimming test}

Forced swimming tests were performed on mice before surgery, during the second week after surgery and on day 5 after BoNT/A injection. Mice were placed in a glass bucket $(18 \mathrm{~cm}$ in diameter and $30 \mathrm{~cm}$ in height) filled with $10 \mathrm{~cm}$ high water at a temperature of $23^{\circ} \mathrm{C} \pm$ $1^{\circ} \mathrm{C}$. Mice were left in the water for 6 minutes to measure the immobility time of each mouse. Immobility was defined as the absence of any active movement of the mice, except for the small movements required to keep them afloat on the water surface.

\section{Western blot analysis}

On day 5 after BoNT/A injection, the mice were under deep anesthesia with $4 \%$ chloral hydrate $(10 \mathrm{ml} / \mathrm{kg}$, i.p.), intracardiac perfusion was performed with $0.9 \%$ saline. The bilateral TNC were quickly removed and then frozen in liquid nitrogen. For further western blot analysis, samples were homogenized in protein lysis buffer containing phosphatase inhibitor and protease inhibitor proteolytic buffer for total protein extraction assays. Protein concentrations were determined by Pierce BCA protein assay (Thermo). After concentration determination and denaturation, protein samples $(30 \mu \mathrm{g})$ were separated on SDS-PAGE gels and electrotransferred onto nitrocellulose membranes. After sealing with $5 \%$ fat-free milk, PVDF membranes were incubated with primary polyclonal antiMyD88 (rabbit, 1:1000; abcam). For loading control, PVDF membranes were incubated with $\beta$-tubulin antibody (mouse, 1:2000, Vazyme, Nanjing, China) at $4{ }^{\circ} \mathrm{C}$ overnight. The washed blots were incubated with horseradish peroxidase-conjugated goat anti-rabbit and goat anti-mouse IgG secondary antibodies (1:2000, Vazyme, Nanjing, China) for $1 \mathrm{~h}$ at room temperature. The washed protein bands were developed using ultrasensitive ECL chemiluminescence kit (NCMECL Ultra) and analyzed for grayscale values as indicated. The ratio between MyD88 and $\beta$-tubulin was calculated and then normalized to the control measurements. Data from four mice were used for statistical analysis.

\section{Quantitative real-time polymerase chain reaction}

Total RNA was isolated from frozen tissues by guanidinium isothiocyanate-phenol extraction and quantified by measuring absorbance at $260 \mathrm{~nm}$ and $280 \mathrm{~nm} .1 \mu \mathrm{g}$ of total RNA was used for reverse transcription. TLRs, inflammatory factors and cell marker mRNA of TNC were quantified by qPCR (prism 7500; Applied Biosystems, Foster, California). The expression of the relevant mRNAs were normalized by the expression of glyceraldehyde- 3 phosphate dehydrogenase mRNA. The following table shows the primer sequences used for qPCR [19]. Data from four mice were used for statistical analysis. The primer sequences used in the RT-qPCR are shown in Table 2.

\section{Immunofluorescence (IF)}

On day 5 after BoNT/A injection, the mice were completely anesthetized with $4 \%$ chloral hydrate $(10 \mathrm{ml} / \mathrm{kg}$, i.p.) and then perfused with sterile saline throughout the body, followed by fixation with $4 \%$ paraformaldehyde. After fixation, the segment where the mouse TNC was located was taken and the same fixative was left overnight. After sucrose gradient dehydration, the tissues were embedded with OCT tissue embedding agent and frozen at $-80{ }^{\circ} \mathrm{C}$ in the refrigerator. The embedded 
Table 2 The primers used in RT-qPCR

\begin{tabular}{|c|c|c|c|}
\hline & Forward primers & Reverse primers & Accession No. \\
\hline Gapdh & TTGATGGCAACAATCTCCAC & CGTCCCGTAGACAAAATGGT & NM_001001303 \\
\hline TIr1 & ATGTGAGCTGAGGGACTTTG & GGATAGTGGAGACATGTGGAAG & NM_030682 \\
\hline TIr2 & ACCAAGATCCAGAAGAGCCA & CATCACCGGTCAGAAAACAA & NM_011905 \\
\hline TIr3 & GCGTTGCGAAGTGAAGAACT & TTCAAGAGGAGGGCGAATAA & NM_126166 \\
\hline Tlr4 & TTCAGAACTTCAGTGGCTGG & TGTTAGTCCAGAGAAACTTCCTG & NM_021297 \\
\hline Tlr5 & GCAGGATCATGGCATGTCAAC & ATCTGGGTGAGGTTACAGCCT & NM_016928 \\
\hline TIr6 & CCAAGAACAAAAGCCCTGAG & TGTTTGCAACCGATTGTGT & NM_011604 \\
\hline TIr7 & GATGTCCTTGGCTCCCTTC & TTTGTCTCTTCCGTGTCCAC & NM_133211 \\
\hline TIr8 & CGTITACCTTCCTITGTCTATAGAAC & CGTCACAAGGATAGCTTCTGG & NM_133212 \\
\hline Tlr9 & AACCGCCACTTCTATAACCAG & GTAAGACAGAGCAAGGCAGG & NM_031178 \\
\hline TIr11 & CAGGCTGGGATTGCTCATC & CCAGTCAAGGTAAGGCTCAC & NM_205819 \\
\hline TIr12 & GCTCTGATTCCTCTGGTGTAG & AGAATGTGAAATAGCGGGAGAC & NM_205823 \\
\hline TIr13 & GGAGCGCCTTGATCTAACTAACA & TCAGGTGGGTCAGAGAAACCA & NM_205820 \\
\hline $\mid L-1 \beta$ & AGAGCATCCAGCTTCAAATCTC & CAGTTGTCTAATGGGAACGTCA & NM_008361.4 \\
\hline TNF-a & AGCCGATGGGTTGTACCTTG & TTGGGCAGATTGACCTCAGC & NM_001278601.1 \\
\hline IL-6 & TCAGGAAATTTGCCTATTGAAAA & CCAGCTTATCTGTTAGGAGAGCA & NM_031168.2 \\
\hline c-Fos & GACAGCCTTTCCTACTACCA & САTCTTATTCCTITCCCTTCG & XM_021201871.2 \\
\hline $\mathrm{F} 4 / 80$ & ATGGACAAACCAACTTTCAAGGC & GCAGACTGAGTTAGGACCACAA & XM_036160297.1 \\
\hline GFAP & GAATCGCTGGAGGAGGAGAT & GCCACTGCCTCGTATTGAGT & XM_030245571.2 \\
\hline CD11b & GACATGGACGCTGATGGCAATAC & CGAGGCAAGGGACACACTGAC & NM_008401.2 \\
\hline
\end{tabular}

tissues were sectioned ( $20 \mu \mathrm{m}$ thickness) and processed on a cryostat microtome (CM 1950; Leica Microsystems, Wetzlar, Germany). Immunohistochemistry was performed as we described previously [20]. Briefly, sections were incubated with $5 \%$ goat serum and incubated overnight at $4{ }^{\circ} \mathrm{C}$ with primary anti IBA1 antibody (rabbit, 1 : 500, Wako) and anti TLR2 antibody (mouse, 1:100, abcam). Then after washing away the first antibody, sections were incubated with FITC and Cy3 conjugated secondary antibodies for $1 \mathrm{~h}$ at room temperature. Immunostained sections were examined under a ZEISS fluorescence microscope (Carl Zeiss OPMI Pentero, Germany), images were taken and the sections were examined with NIH ImageJ software (NIH, Bethesda, MD) for analysis.

\section{Statistical analysis}

The data was analyzed using Graph Prism 6 (Graph Pad, La Jolla, CA). Shapiro-Wilk test was used to test normality of the data. All values were presented as mean \pm SEM. Unpaired Student's t-test was used to compare two groups. Two-Way Repeated Measures ANOVA with post-hoc Bonferroni test was performed for multiple comparisons. Differences were considered statistically significant at $P<0.05$.

\section{Results}

A modified TN model was established by chronic constriction injury $(\mathrm{CCl})$ of the unilateral distal infraorbital nerve (dloN) in mice

We used von Frey filaments to evaluate mechanical pain sensitivity in the bilateral whisker pad of mice before surgery by measurement of the frequency of positive reactions to the filaments in mice. One week after dIoNCCI surgery, the bilateral mechanical pain sensitivity in the whisker pad was detected. Compared to that of the sham animals, dIoN-CCI mice showed a significant mechanical pain hypersensitivity on the injured side at $0.008 \mathrm{~g}$ mechanical stimulus intensity $\left(\mathrm{F}_{\text {time }}(5,35)=69\right.$, $P<0.0001 ; \mathrm{F}_{\text {surgery }(1,7)}=541.3, P<0.0001 ; \mathrm{F}_{\text {time } \times \operatorname{surgery}(5,}$ $35)=37.62, P<0.0001$; Fig. 1a), and the similar results were also observed on the contralateral side $\left(\mathrm{F}_{\text {time }}(5\right.$, $35)=58.97, P<0.0001 ; \mathrm{F}_{\text {surgery }(1,7)}=203.8, P<0.0001$; $\mathrm{F}_{\text {time } \times \operatorname{surgery}(5,35)}=27.08, P<0.0001$; Fig. $\left.1 \mathrm{~b}\right)$. At $0.02 \mathrm{~g}$ mechanical stimulation intensity, the frequency of positive responses to filaments had the same trend as the $0.008 \mathrm{~g}$ stimulation intensity (ipsilateral: $F_{\text {time }(5,35)}=$ 36.46, $P<0.0001 ; \mathrm{F}_{\text {surgery }(1,7)}=453.6, P<0.0001 ; \mathrm{F}_{\text {time } \times}$ surgery(5,35) $=21.78, P<0.0001$; Fig. 1c; contralateral: $\mathrm{F}_{\text {time }(5,35)}=83.26, P<0.0001 ; \mathrm{F}_{\text {surgery }(1,7)}=468, P<$ $0.0001 ; F_{\text {time } \times \operatorname{surgery}(5,35)}=34.68, P<0.0001 ;$ Fig. 1d). Thus, these data indicated that a modified mouse model 

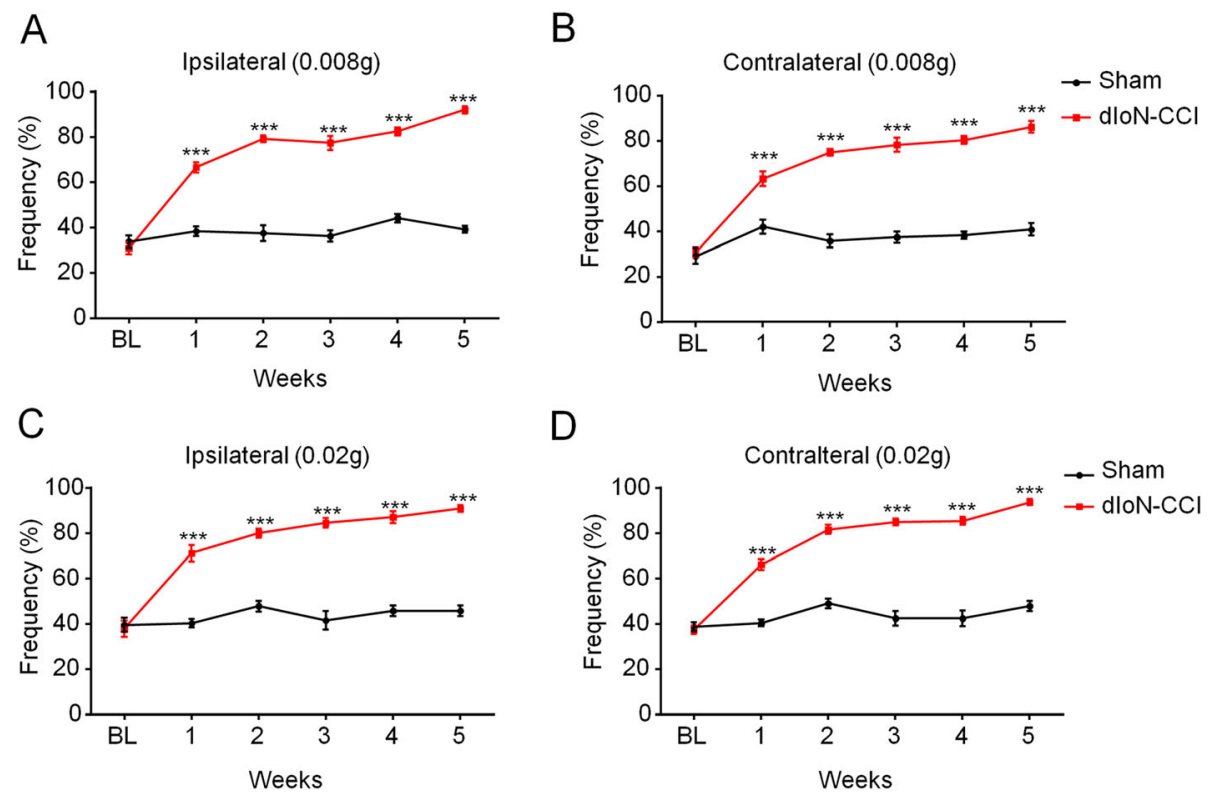

Fig. 1 Bilateral mechanical pain hypersensitivity during 5 weeks following dloN-CCl surgery in mice. a-b At $0.008 \mathrm{~g}$ stimulus intensity, mechanical pain hypersensitivity in dloN-CCl mice was observed in the ipsilateral (a) and contralateral side (b) to dloN-CCl surgery compared with that of sham mice. c-d At $0.02 \mathrm{~g}$ stimulus intensity, mechanical pain hypersensitivity in dloN-CCl mice was observed in the ipsilateral (c) and contralateral side $(\mathbf{d})$ to dloN-CCl surgery compared with that of sham mice. Data was expressed as Mean \pm SEM. $\left(n=8\right.$ each group; ${ }^{* * *} P<0.001$ compared with the sham group, two-way ANOVA with post-hoc Bonferroni test). dloN-CCl, chronic constriction injury of distal infraorbital nerve; BL, baseline

of trigeminal neuralgia (TN) was successfully established and we found that unilateral ligation of the distal infraorbital nerve caused bilateral mechanical pain hypersensitivity in the whisker pad lasting for at least 5 weeks.

\section{Ipsilateral facial subcutaneous injection of BoNT/A attenuated bilateral mechanical pain hypersensitivity caused by dloN-CCl surgery in mice}

After establishment of TN mouse model, dIoN-CCI mice were divided into two groups, $\mathrm{TN}+$ vehicle group and $\mathrm{TN}+\mathrm{BoNT} / \mathrm{A}$ group. We subcutaneously injected BoNT/A into the ipsilateral whisker pad, and assessed the analgesic effect of BoNT/A by examining the bilateral mechanical pain hypersensitivity in the whisker pad in dIoN-CCI mice. At $0.008 \mathrm{~g}$ mechanical stimulation intensity, the analgesic effect of BoNT/A injection appeared after $1 \mathrm{~h}$ injection into the ipsilateral side, and the analgesic effect was still detectable on the fifth day after BoNT/A injection. The analgesic effect of BoNT/A lasted for about 9 days. Two weeks after the analgesic effect had worn off, a second injections of BoNT/A was performed to test the analgesic effects of repeated injection of BoNT/A in mice. The data showed a second facial injection of BoNT/A produced similar analgesic effect in the ipsilateral side of dIoN-CCI surgery in mice $\left(\mathrm{F}_{\text {time }(14,98)}=28.3, P<0.0001 ; \mathrm{F}_{\text {treatment }(1,7)}=134.3, P<\right.$ $0.0001 ; F_{\text {time } \times \text { treatment }(14,98)}=27.11, P<0.0001$; Fig. $\left.2 \mathrm{a}\right)$.
The analgesic effect was also evident on the contralateral side of dIoN-CCI surgery in mice $\left(F_{\text {time }(14,98)}=26.08\right.$, $P<0.0001 ; \mathrm{F}_{\text {treatment }(1,7)}=49.41, P<0.0001 ; \mathrm{F}_{\text {time } \times \text { treat }}$ ment(14, 98) $=15.43, P<0.0001$; Fig. $2 b)$. The similar analgesic effect at $0.02 \mathrm{~g}$ mechanical stimulation intensity had been observed on the bilateral sides of dIoN-CCI surgery in mice (ipsilateral: $\mathrm{F}_{\text {time }(14,98)}=25.59, P<$ $0.0001 ; \mathrm{F}_{\text {treatment }(1,7)}=213.4, P<0.0001 ; \mathrm{F}_{\text {time }} \times$ treatment $(14$, 98) $=24.26, P<0.0001$; Fig. $2 \mathrm{c}$; contralateral: $F_{\text {time }}(14$, 98) $=23.95, P<0.0001 ; F_{\text {treatment }(1,7)}=55.33, P<0.0001$; $\mathrm{F}_{\text {time } \times \text { treatment }(14,98)}=27.75, P<0.0001$; Fig. $\left.2 \mathrm{~d}\right)$. Thus, these results suggested that unilateral subcutaneous injection of BoNT/A is an effective and reproducible treatment for the relief of trigeminal neuropathic pain, including mirror image pain in mice.

Facial injection BoNT/A relieved anxiety-like behavior (but not depression-like behavior) induced by dloN-CCI surgery in mice

On day 14 after dIoN-CCI surgery in mice, we performed the open field test. We found that dIoN-CCI mice took less time to enter the intermediate area than the sham group. On day 5 after BoNT/A injection (day 19 after dIoN-CCI surgery), the BoNT/A-treated dIoN$\mathrm{CCI}$ mice spent more time in the center area compared to the vehicle-treated group (on day 14 before BoNT/A injection: sham vs. dIoN-CCI, $\mathrm{t}_{9}=3.145, P=0.0118$; 

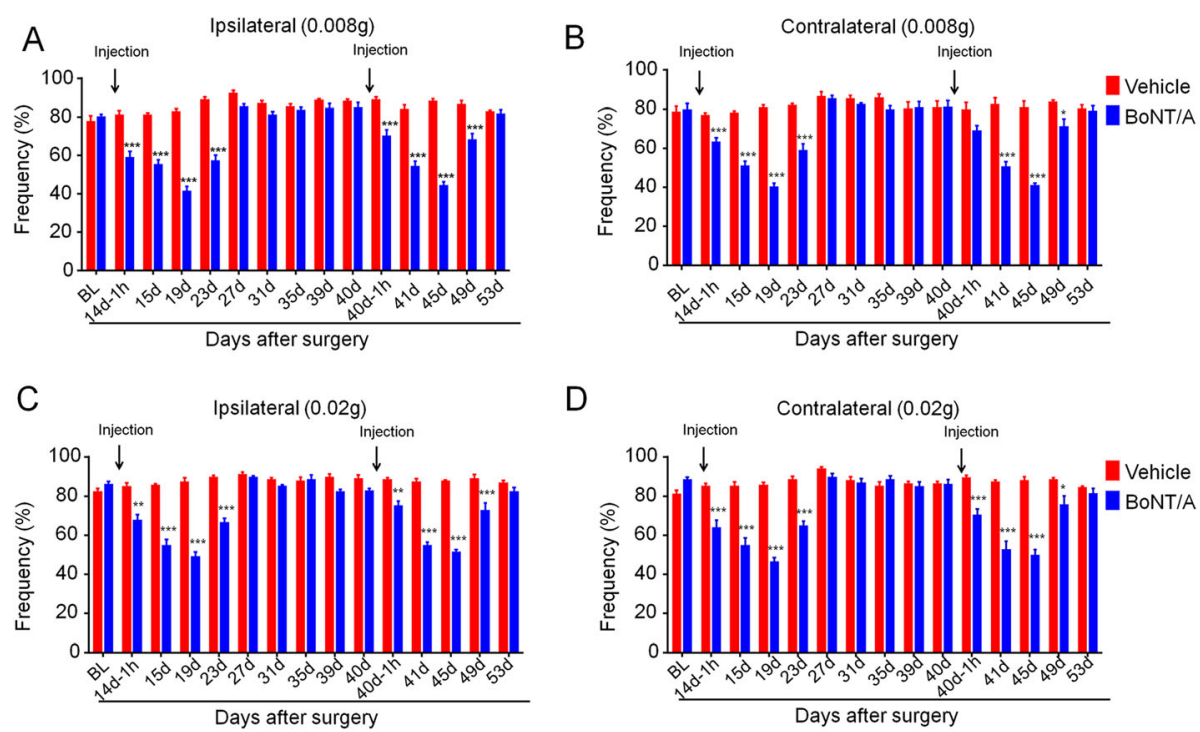

Fig. 2 Unilateral facial injection of BoNT/A attenuated bilateral mechanical pain hypersensitivity induced by dloN-CCl surgery in mice. Black arrow represented the time of BoNT/A injection in mice. a-b At $0.008 \mathrm{~g}$ von Frey stimulation intensity, facial injection of BoNT/A significantly attenuated ipsilateral (a) and contralateral (b) mechanical pain hypersensitivity induced by dloN-CCl surgery in mice, which lasted approximately 9 days, compared to the vehicle group. After 2 weeks, a second injections of BoNT/A showed the similar analgesic effect. c-d At $0.02 \mathrm{~g}$ von Frey stimulation intensity, facial injection of BoNT/A significantly attenuated ipsilateral (a) and contralateral (b) mechanical pain hypersensitivity induced by dloN-CCl surgery in mice, which lasted approximately 9 days, compared to the vehicle group. After 2 weeks, a second injections of BoNT/A showed the similar analgesic effect. Data was expressed as Mean \pm SEM. $\left(n=8\right.$ each group, ${ }^{*} P<0.05,{ }^{* *} P<0.01,{ }^{* * *} P<0.001$, two-way ANOVA with post-hoc Bonferroni test). dloN-CCl, chronic constriction injury of distal infraorbital nerve; BoNT/A botulinum toxin A; BL, baseline

dIoN-CCI vs. dIoN-CCI + BoNT/A, $t_{9}=3.368, \quad P=$ 0.0083; on day 5 after BoNT/A injection: sham vs. dIoNCCI, $\mathrm{t}_{10}=5.218, P=0.0004$; dIoN-CCI vs. dIoN-CCI + BoNT/A, $\mathrm{t}_{9}=2.484, P=0.0348$; Fig. 3a). Additionally, on day 14 after dIoN-CCI surgery, dIoN-CCI mice excreted at a higher rate than the sham group. On day 5 after BoNT/A injection (day 19 after dIoN-CCI surgery), the excretion rate of mice was reduced compared to the vehicle-treated group (on day 14 before BoNT/A injection: sham vs. dIoN-CCI + vehicle, $\mathrm{t}_{10}=7.071, P<$ 0.0001; dIoN-CCI + vehicle vs. dIoN-CCI + BoNT/A, $\mathrm{t}_{10}=4.227, P=0.0018$; on day 5 after BoNT/A injection: $\mathrm{dIoN}-\mathrm{CCI}+$ vehicle vs. dIoN-CCI $+\mathrm{BoNT} / \mathrm{A}, \mathrm{t}_{10}=3.422$, $P=0.0065$; Fig. $3 \mathrm{~b}$ ). Furthermore, we performed the elevated plus maze experiment on day 5 after BoNT/A injection (day 19 after dIoN-CCI surgery), the mice showed a significant decrease in the proportion of duration into the open arms compared to the vehicletreated group $\left(\mathrm{t}_{11}=2.742 ; P=0.0192 ;\right.$ Fig. $\left.3 \mathrm{c}\right)$. There was a decrease in the percentage of times access to the open arm (sham vs. dIoN-CCI + vehicle, $\mathrm{t}_{10}=2.299, P=$ 0.0443; dIoN-CCI + vehicle vs. dIoN-CCI + BoNT/A, $\mathrm{t}_{9}=2.320, P=0.0455$; Fig. $\left.3 \mathrm{~d}\right)$. We also employed forced swimming experiments to study the depression-like behavior in mice. We found no significant differences in the duration of immobility in the tested three groups of mice (Fig. 3e). Additionally, there was also no significant change in the body weight of the three groups of mice during the experiment (Fig. 3f). These results suggested that TN induced anxiety-like behavior (rather than depression-like behavior) in mice, and facial injection of BoNT/A significantly relieved anxiety-like behaviors induced by TN in mice.

Facial injection of BoNT/A down-regulated the expression of toll-like receptors (TLRs) in the TNC in dloN-CCl surgery mice

To investigate the role of TLRs in the pathogenesis of TN, we first examined the expression of TLRs in the TNC in wild-type (WT) male mice. We found that all TLRs were expressed in the TNC with different expression levels in naive mice (Fig. 4a). We then examined the expression of TLRs in the bilateral TNC of the tested three groups. In the ipsilateral TNC, dIoN-CCI surgery caused a significant increase in the mRNA expression of TLR2 and TLR5, a significant decrease in the mRNA expression of TLR11 in mice (sham vs. dIoN-CCI + vehicle, TLR2: $\mathrm{t}_{6}=6.307, P=$ 0.0007; TLR5: $\mathrm{t}_{6}=7.584, P=0.0003$; TLR11: $\mathrm{t}_{6}=10.8, P<$ 0.0001). On day 5 after BoNT/A injection (day 19 after dIoN-CCI surgery), BoNT/A-treated mice showed a significant decrease in the mRNA expression of TLR1, TLR2, TLR4, TLR5 and TLR8, compared with vehicle-treated mice (dIoN-CCI + vehicle vs. dIoN-CCI + BoNT/A, TLR1: $\mathrm{t}_{6}=3.393, P=0.0146 ;$ TLR2: $\mathrm{t}_{6}=6.058, P=0.0009 ;$ TLR4: $\mathrm{t}_{6}=8.721, P=0.0001 ;$ TLR5: $\mathrm{t}_{6}=6.897, P=0.0005 ;$ TLR8: $\mathrm{t}_{6}=5.766, P=0.0012$; Fig. $\left.4 \mathrm{~b}\right)$. On the contralateral side, 
A

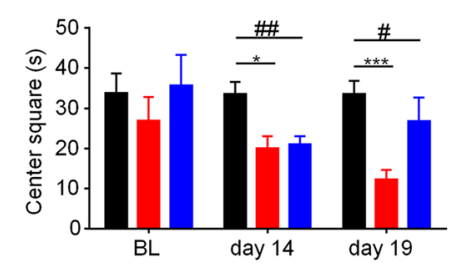

$c$

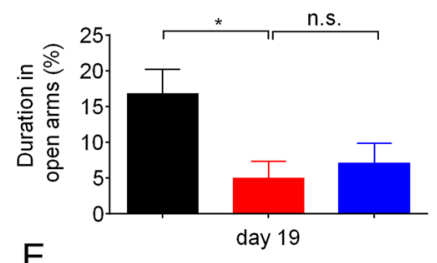

$\mathrm{E}$

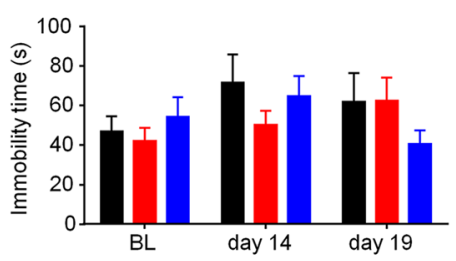

B

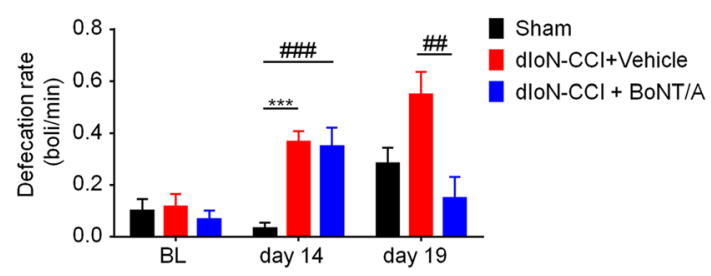

D

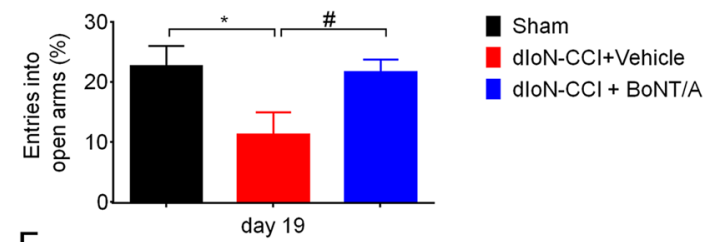

$\mathrm{F}$

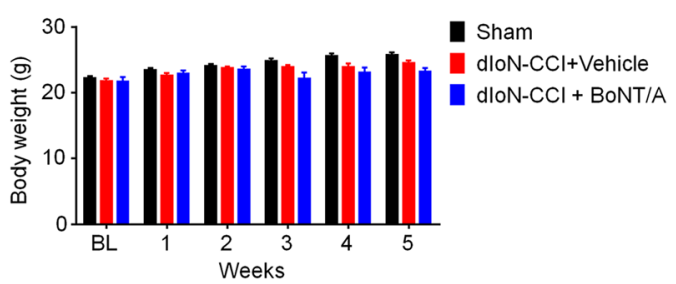

Fig. 3 Facial injection of BoNT/A attenuated dloN-CCl surgery-induced anxiety-like behavior, but not depression-like behavior, in mice. On day 14 following dloN-CCl surgery, BoNT/A was injected in dloN-CCl mice. a In open field test, on day 5 after BoNT/A injection (day 19 following dloN$\mathrm{CCl}$ surgery), BoNT/A treatment significantly increase the time in the center area compared with that of vehicle group. b On day 5 after BoNT/A injection (day 19 following dloN-CCl surgery), BoNT/A treatment significantly decrease the excretion rate compared with that of vehicle group. cd In an elevated plus maze, on day 5 after BoNT/A injection (day 19 following dloN-CCl surgery), BoNT/A treatment had no significant effect on the percentage of duration of open arms compared with that of vehicle group (c). BoNT/A treatment significantly increased the percentage of entries into the open arm in an elevated plus maze than that of vehicle group (d). e-f There was no significant change in the duration of the immobility time in FST test $(\mathbf{e})$, the body weight $(\mathbf{f})$ among the three groups during the experiment. Data was expressed as Mean \pm SEM. $(n=8$ each group, n.s., no significance, ${ }^{*} P<0.05,{ }^{* * *} P<0.001$ compared with the sham group. ${ }^{\#} P<0.05,{ }^{\# \#} P<0.01$, \#\#\# $P<0.001$ compared with the dloN-CCl group, unpaired Student's t-test). dloN-CCl, chronic constriction injury of distal infraorbital nerve; BoNT/A, botulinum toxin A; BL, baseline; FST, forced swimming test

the dIoN-CCI surgery and sham groups showed no significant change in the mRNA expression of TLRs. However, the expression TLR1, TLR4 and TLR8 were significantly decreased on day 5 after BoNT/A injection (day 19 after dIoN-CCI surgery) compared with vehicle-treated mice (TLR1: $\mathrm{t}_{6}=6.614, P=0.0006$; TLR4: $\mathrm{t}_{6}=6.963, P=0.0004$; TLR8: $t_{6}=2.7, P=0.0356$; Fig. $4 \mathrm{c}$ ). Thus, these results suggested that changes of TLRs expression in the TNC were involved in the pathogenesis of TN and BoNT/A treatment significantly down-regulated the mRNA expression of TLRs, particularly TLR2 and TLR5.

\section{TLR2 deficiency alleviates bilateral mechanical pain hypersensitivity in the dloN-CCl mice}

Subsequently, we employed $T l r 2^{-/-}$mice to investigate the role of TLR2 on the development of persistent pain in the dIoN-CCI mouse model. The results showed that at $0.008 \mathrm{~g}$ mechanical stimulation intensity, $T l r 2^{-/-}$mice had a significant lower positive response rate on the ipsilateral side $\left(\mathrm{F}_{\text {time }}(5\right.$, $54)=57.89, P<0.0001 ; F_{\text {genotype }(1,54)}=112, P<$ $0.0001 ; \quad F_{\text {time } \times \text { genotype }}(5,54)=1.857, \quad P=0.1174$; Fig. 5a) and contralateral side of dIoN-CCI surgery $\left(\mathrm{F}_{\text {time }(5,54)}=114.9, P<0.0001 ; \mathrm{F}_{\text {genotype }}(1,54)=\right.$ $149.1, P<0.0001 ; \mathrm{F}_{\text {time }} \times$ genotype $(5,54)=5.614, P=$ 0.0003; Fig. 5b) compared with wild-type mice. At $0.02 \mathrm{~g}$ stimulation intensity, $T l r 2^{-/-}$mice showed a significantly lower positive response rate on the ipsilateral side $\left(\mathrm{F}_{\text {time }(5,54)}=104.9, P<0.0001 ; \mathrm{F}_{\text {genotype }}\right.$ $(1,54)=136.6, P<0.0001 ; F_{\text {time }} \times$ genotype $(5,54)=2.345$, $P=0.0535$; Fig. 5c) and contralateral side of dIoNCCI surgery $\left(\mathrm{F}_{\text {time }}(5,54)=60.08, P<0.0001 ; F_{\text {genotype }}\right.$ $(1,54)=64.59, P<0.0001 ; \mathrm{F}_{\text {time }} \times$ genotype $(5,54)=$ $0.8623, P=0.5124$; Fig. 5 d) compared with wild-type mice. Thus, these results suggested that TLR2 deletion alleviates dIoN-CCI induced bilateral mechanical 


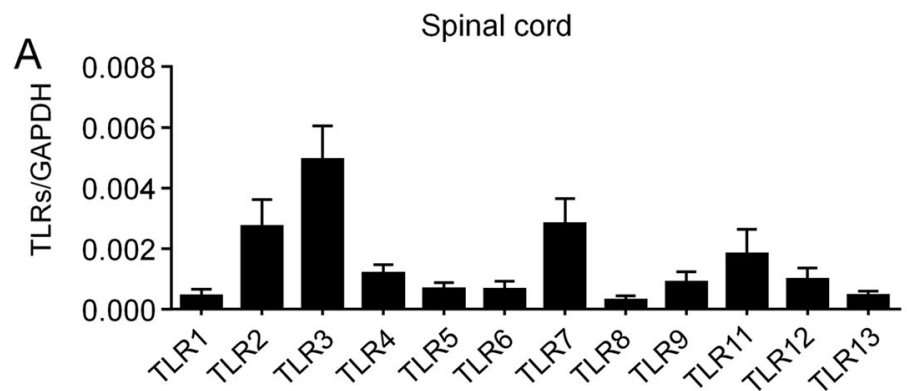

B

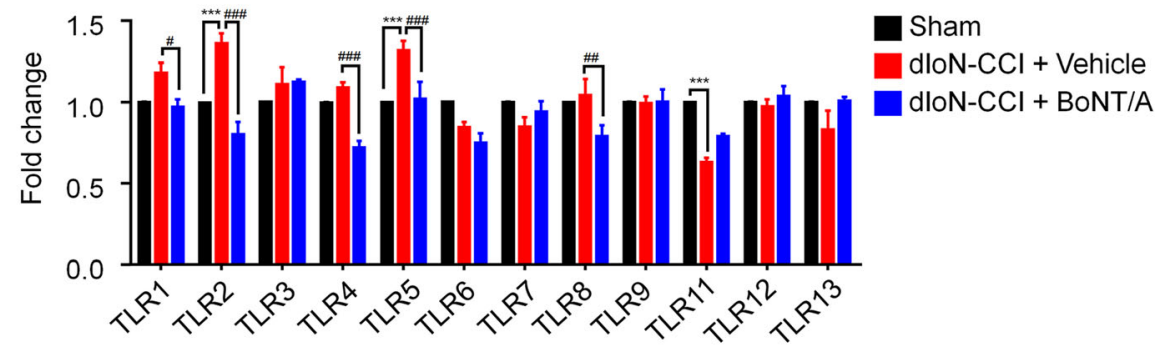

C

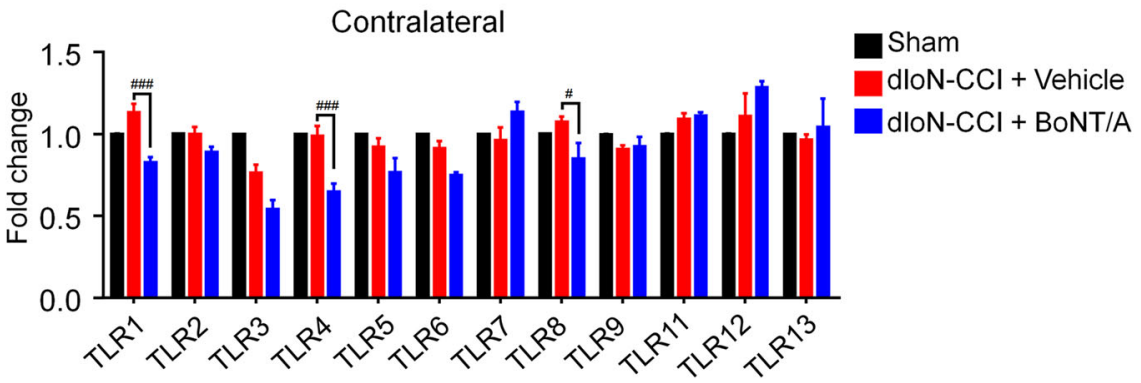

Fig. 4 Expression of TLRs in bilateral TNC using RT-qPCR. a RT-qPCR analyzed the expression levels of TLRs in wild-type mice. GAPDH was used as internal control $(n=6)$. b Relative expression of TLRs in the ipsilateral TNC on day 5 after BoNT/A injection (day 19 following dloN-CCl surgery) among the three groups. There was an increase in TLR2 and TLR5, a decrease in TLR11 in the dloN-CCl group compared with the sham group, while there was a decrease in TLR1, TLR2, TLR4, TLR5 and TLR8 expression after BoNT/A injection. c Relative expression of TLRs in the contralateral TNC on day 5 after BoNT/A injection (day 19 following dloN-CCl surgery) among the three groups. The dloN-CCl and sham group showed no significant change in TLRs expression, while TLR1, TLR4 and TLR8 expression decreased after BoNT/A injection. Data was expressed as Mean \pm SEM. ( $n=4$ each group, ${ }^{* * *} P<0.001$ compared with the sham group. ${ }^{\#} P<0.05,{ }^{\# \#} P<0.01$, ${ }^{\# \# \# ~} P<0.001$ compared with the dloN-CCl group, unpaired Student's t-test). TLRs, toll-like receptors; TNC, trigeminal nucleus caudalis; dloN-CCl, chronic constriction injury of distal infraorbital nerve; BoNT/A, botulinum toxin-A; BL, baseline

pain hypersensitivity compared with that of wild-type mice.

The inhibitory effects of BoNT/A treatment on the mRNA expression of glia activation markers and proinflammatory mediators in the TNC of the dloN-CCI mice

We subsequently investigated the expression changes of glia and proinflammatory mediators in the TNC induced by $\mathrm{TN}$ and the possible effects of BoNT/A injection on these changes. On the ipsilateral side of dIoN-CCI surgery, the mRNA expression of markers F4/80 (macrophage or microglia marker), CD11b (microglia marker) and c-Fos (neuron activation marker) were significantly increased in the dIoN-CCI group compared with sham control (sham vs. dIoN-CCI + vehicle, F480: $t_{6}=2.965$, $P=0.0351$; CD11b: $t_{6}=0.3945, P=0.0076$; c-Fos: $t_{6}=$ 4.707, $P=0.0033$ ), and BoNT/A treatment significantly decrease these markers on day 5 after BoNT/A injection (day 19 after dIoN-CCI surgery) (dIoN-CCI + vehicle vs. $\mathrm{dIoN}-\mathrm{CCI}+\mathrm{BoNT} / \mathrm{A}, \quad \mathrm{F} 4 / 80: \quad \mathrm{t}_{6}=4.799, \quad P=0.003$; CD11b: $\mathrm{t}_{6}=4.994, P=0.0025 ; \mathrm{c}-$ Fos: $\mathrm{t}_{6}=5862, P=$ 0.0011; Fig. 6a). On the contralateral side of dIoN-CCI surgery, the mRNA expression of c-Fos was significantly increased in the dIoN-CCI group compared with sham group (sham vs. dIoN-CCI: $t_{6}=4.022, P=0.0069$ ), and the upregulation of mRNA expression of c-Fos was significantly decreased in BoNT/A-treated animals 

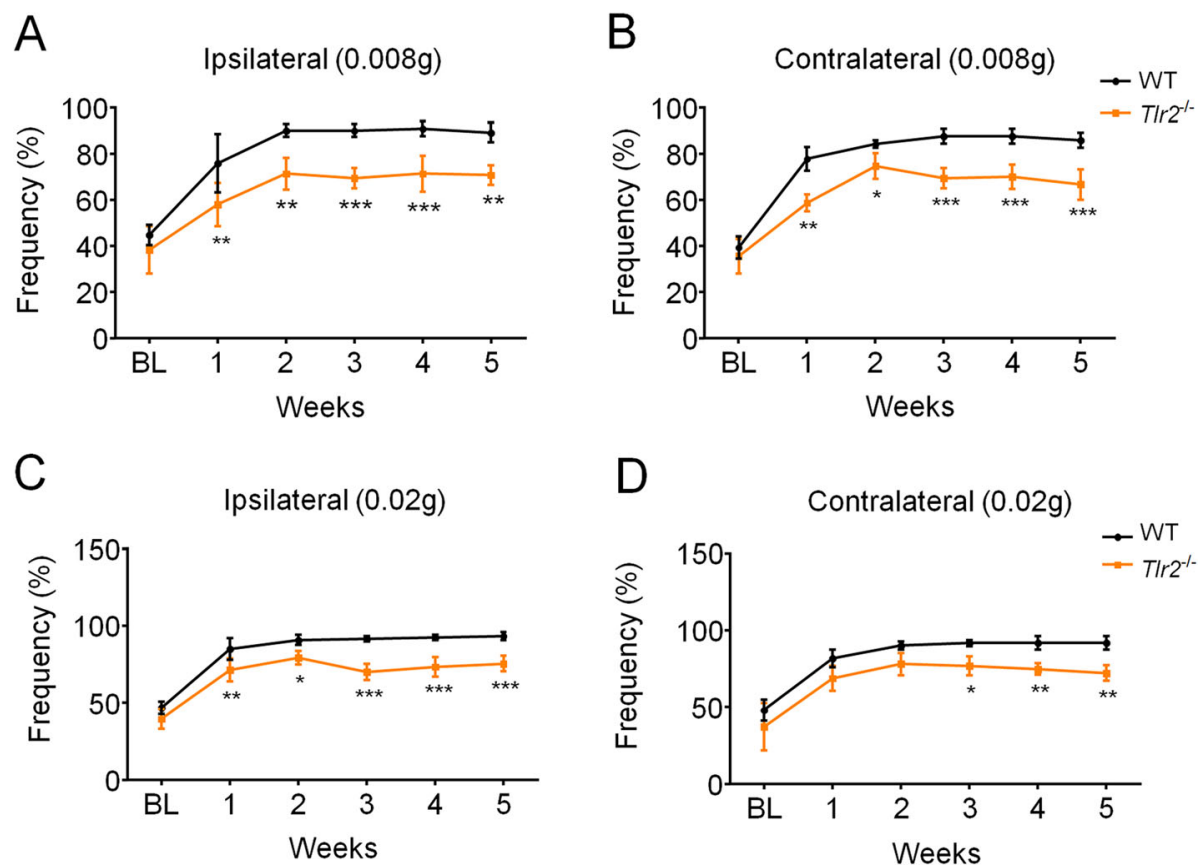

Fig. 5 Bilateral mechanical pain hypersensitivity following dloN-CCl surgery in the wide-type and T/r2 ${ }^{-/-}$mice. a-b At $0.008 \mathrm{~g}$ stimulus intensity, ipsilateral (a) and contralateral (b) mechanical pain hypersensitivity induced by dloN-CCl group was significantly inhibited in the $T / r^{2 /-}$ mice compared with that of wild-type mice. c-d At $0.02 \mathrm{~g}$ stimulus intensity, ipsilateral (c) and contralateral (d) mechanical pain hypersensitivity induced by dloN-CCl group was significantly inhibited in the T/r $2^{-1-}$ mice compared with that of wild-type mice. Data was expressed as Mean \pm SEM. ( $n=6$ each group, ${ }^{*} P<0.05,{ }^{* *} P<0.01,{ }^{* * *} P<0.001$ compared with the C57 dloN-CCl group, two-way ANOVA with post-hoc Bonferroni test). dloN-CCl, chronic constriction injury of distal infraorbital nerve; BL, baseline

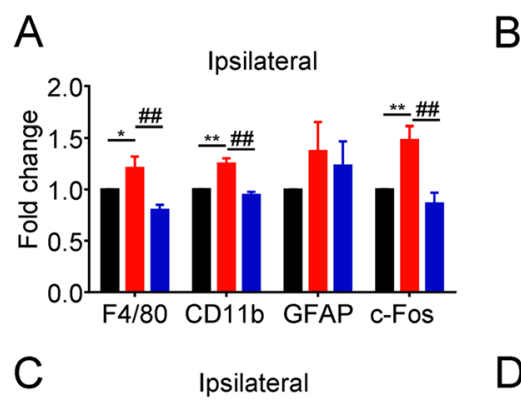

B Contralateral
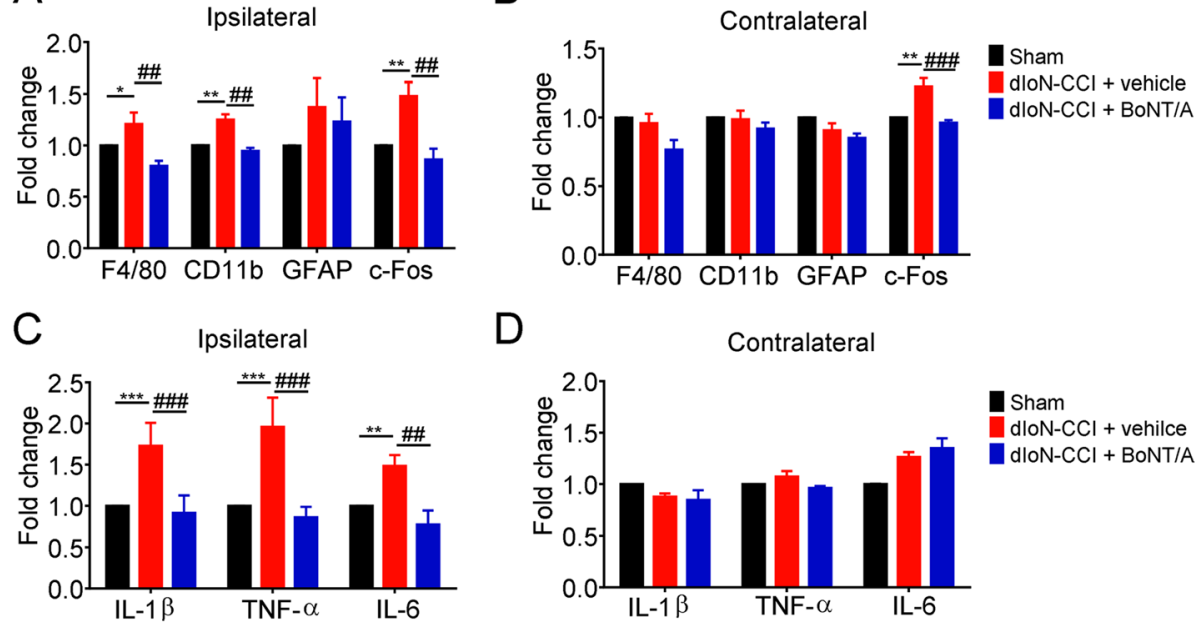

Sham

dloN-CCl + vehilce

dloN-CCl + BoNT/A

Fig. 6 The expression of glia activation markers and proinflammatory factors in bilateral TNC site using RT-qPCR. a On the ipsilateral TNC of dloN$\mathrm{CCl}$ surgery, the mRNA expression of F4/80, CD11b and c-FOS were significantly increased by dloN-CCl surgery, and BoNT/A treatment significantly decreased their up-regulation. $\mathbf{b}$ On the contralateral TNC of dloN-CCl surgery, only the mRNA expression of c-FOS was increased by dloN-CCl surgery, and BoNT/A treatment significantly decreased the up-regulation of c-FOS. c On the ipsilateral TNC of dloN-CCl surgery, the mRNA expression of pro-inflammatory factors IL-1 $\beta$, TNF- $a$ and IL-6 were significantly elevated by dloN-CCI surgery, and BoNT/A treatment significantly decreased their up-regulation. $\mathbf{d}$ There was no statistically significant difference in the expression of proinflammatory factors on the contralateral TNC of dloN-CCl surgery. Data was expressed as Mean \pm SEM. $\left(n=4\right.$ each group, ${ }^{*} P<0.05,{ }^{* *} P<0.01,{ }^{* * *} P<0.001$ compared with the sham group. \#\# $P<0.01$, \#\#\# $P<0.001$ compared with the dloN-CCl group, unpaired Student's t-test). TNC, Trigeminal nucleus caudalis; dloN$\mathrm{CCl}$, Chronic constriction injury of distal infraorbital nerve; BoNT/A, Botulinum toxin A 
compared with that of vehicle-treated group (dIoN$\mathrm{CCI}+$ vehicle vs. dIoN-CCI + BoNT/A, $\mathrm{t}_{6}=8.647, P=$ 0.0001 ; Fig. $6 \mathrm{~b}$ ). The mRNA expression of proinflammatory factors IL-1 $\beta$, TNF- $\alpha$ and IL- 6 were significantly elevated in the ipsilateral side in dIoN-CCI surgery group compared with that of sham group (sham vs. dIoNCCI + vehicle: IL-1 $\beta: \mathrm{t}_{6}=8.633, P=0.0001$; TNF- $\alpha: \mathrm{t}_{6}=$ 8.990, $P=0.0001$; IL-6: $\mathrm{t}_{6}=5.629, P=0.0013$ ), and the upregulation of mRNA expression of these proinflammatory factors was significantly decreased on day 5 after BoNT/A injection (day 19 after dIoN-CCI surgery) animals (dIoN-CCI + vehicle vs. dIoN-CCI + BoNT/A, IL$1 \beta: \mathrm{t}_{6}=10.39, P<0.0001 ;$ TNF- $\alpha: \mathrm{t}_{6}=9.878, P<0.0001$; IL-6: $\mathrm{t}_{6}=4.657, P=0.0035$; Fig. $6 \mathrm{c}$ ). However, there was no statistically significant difference in the expression of proinflammatory factors in the contralateral side of dIoN-CCI surgery compared with that of sham animals (Fig. 6d). Together, the results suggested that dIoN-CCI surgery induced microglia activation and neuroinflammation in the TNC on the ipsilateral side of the surgery, and BoNT/A injection inhibited microglia activation and upregulation of proinflammatory factors in mice.
Interestingly, BoNT/A treatment significantly reduced bilateral activation of neurons in the TNC induced by dIoNCCI surgery, which may be associated with analgesic effects of BoNT/A on mirror image pain.

\section{Microglia activation in the ipsilateral TNC induced by dloN-CCl surgery in mice}

We then used IBA-1 (a microglia marker) immunofluorescence staining to investigate microglia activation in the TNC induced by dIoN-CCI surgery and the effects of BoNT/A on it. We showed that in on day 14 after dIoNCCI surgery, there was upregulation of IBA-1-positive cells in the ipsilateral TNC of dIoN-CCI surgery, indicating ipsilateral activation in microglia induced by dIoNCCI surgery in mice (Fig. 7a). There was no obvious changes of IBA-1 expression in the contralateral TNC of dIoN-CCI surgery group (Fig. 7a). Quantitative analysis of the intensity of IBA-1 immunofluorescence showed in the dIoN-CCI surgery mice, higher fluorescence intensity was found in the ipsilateral TNC than that of the contralateral side of the surgery $\left(\mathrm{t}_{4}=4.332, P=0.0123\right.$; Fig. 7b). By using immunostaining, we also compared
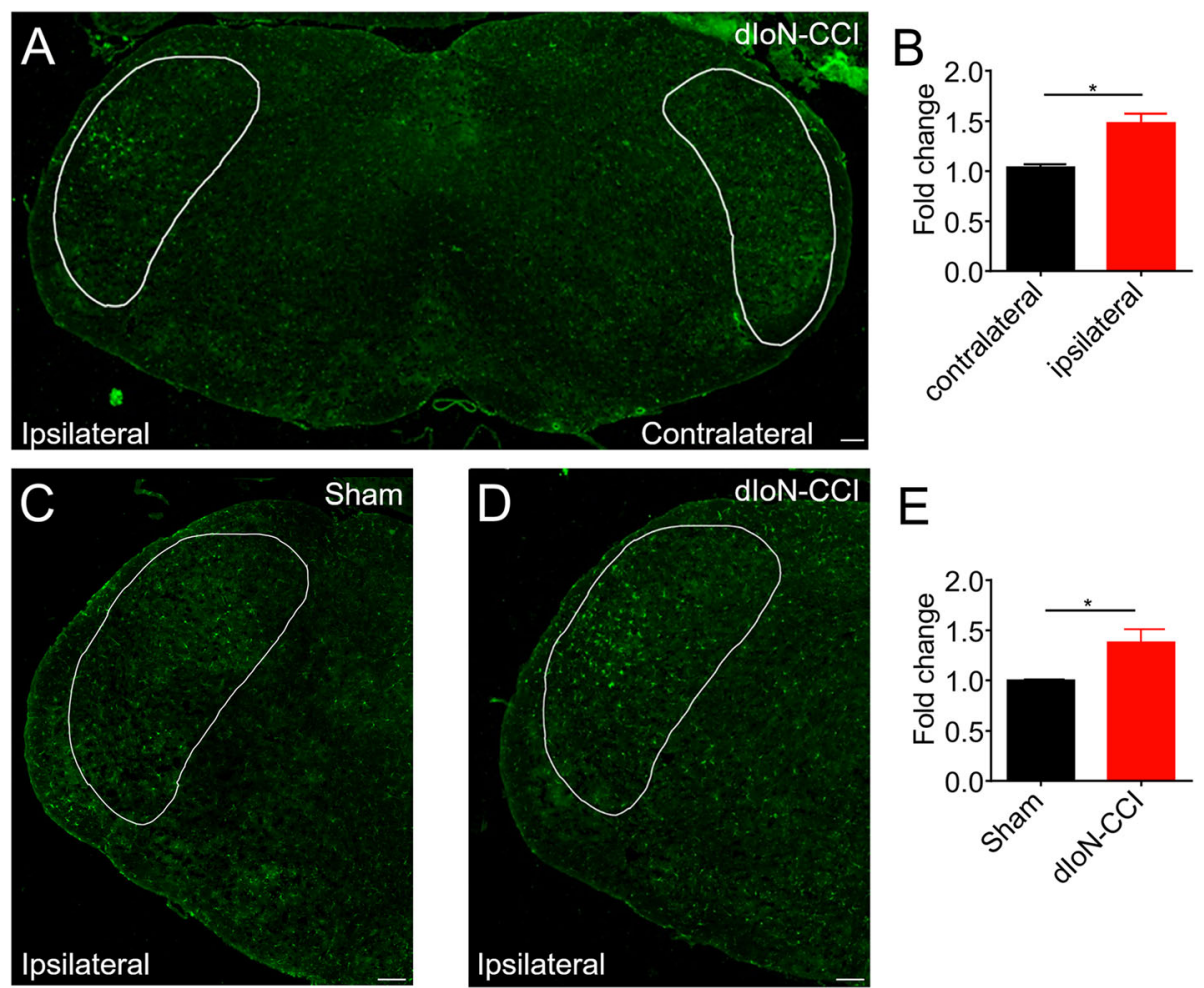

Fig. 7 Immunostaining analysis showed the protein expression of IBA-1 in the TNC site (the area circled by the white solid circle). a Upregulation of IBA-1 expression in the ipsilateral TNC induced by dloN-CCl surgery in mice. $\mathbf{b}$ Quantitative analysis of IBA-1 immunofluorescence intensity in the ipsilateral and contralateral TNC site in the dloN-CCl group, as fold of contralateral side to surgery. $\mathbf{c}$ Expression of IBA-1 in the ipsilateral TNC in the sham mice. $\mathbf{d}$ Upregulation of IBA-1 expression in the ipsilateral TNC in the dloN-CCl surgery mice. e Quantitative analysis of IBA-1 immunofluorescence intensity in the ipsilateral TNC between sham and dloN-CCI group. Data was expressed as Mean \pm SEM. ( $n=3$ each group, scale bar $=100 \mu \mathrm{m},{ }^{*} P<0.05$ compared with the control, unpaired Student's t-test) TNC, trigeminal nucleus caudalis; dloN-CCl, chronic constriction injury of distal infraorbital nerve 

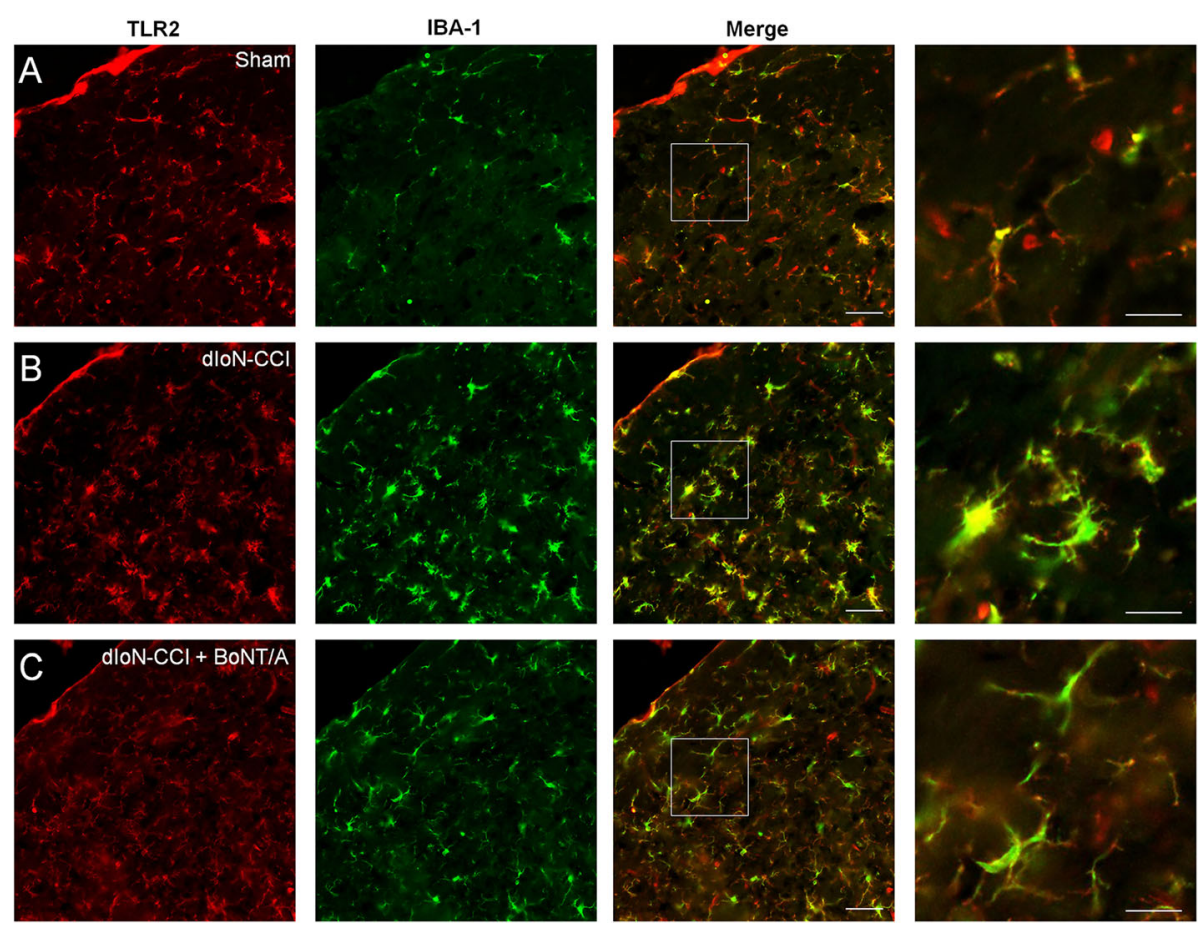

Fig. 8 Double immunostaining of TLR2 and IBA-1 in the ipsilateral TNC induced by dloN-CCl surgery in mice. a Double immunostaining of TLR2 and IBA-1 in the ipsilateral TNC induced in sham animals. $\mathbf{b}$ Double immunostaining of TLR2 and IBA-1 in the ipsilateral TNC in dloN-CCI animals. c On the day 5 following BoNT/A treatment (on day 19 following dloN-CCl surgery), double immunostaining of TLR2 and IBA-1 in the ipsilateral TNC in dloN-CCl + BoNT/A animals. Scale bar $=50 \mu \mathrm{m}$, and the scale bar in the enlarged graph is $20 \mu \mathrm{m}$. TNC, Trigeminal nucleus caudalis; dloN$\mathrm{CCl}$, chronic constriction injury of distal infraorbital nerve; BoNT/A, botulinum toxin A

the microglia activation in the ipsilateral TNC of surgery between the sham and dIoN-CCI surgery group. We found the IBA-1 fluorescence intensity in the ipsilateral side of TNC of dIoN-CCI mice was significantly higher than that of the sham group $\left(t_{4}=2.949, P=0.0420\right.$; Fig. $7 \mathrm{c}-\mathrm{e})$. Thus, the results indicated that ipsilateral microglia activation in the TNC was induced by dIoNCCI surgery compared with that of sham group.

\section{The inhibitory effects of BoNT/A treatment on}

\section{upregulation of the expression of TLR2 and IBA-1 in the} ipsilateral side of dloN-CCl surgery in mice

By using immunofluorescence, the co-localization of IBA-1 and TLR2 at the TNC of the surgery side in all tested three groups was detected. Co-localization of IBA-1 and TLR2 was detected in the sham mice (Fig. 8a). On day 14 after dIoN-CCI surgery in mice, we found the increased expression of both TLR2 and IBA-1 in the activated microglia in the ipsilateral side of dIoN-CCI surgery (Fig. 8b). The upregulation of TLR2 and IBA-1 was significantly suppressed on day 5 after BoNT/A injection (day 19 after dIoN-CCI surgery) in dIoN-CCI mice (Fig. 8c). Thus, these results suggested that microglia activation was inhibited and co-localization of IBA-1 and
TLR2 was reduced after BoNT/A injection in the TNC on the surgical side.

BoNT/A reduced the upregulation of MyD88 expression after dloN-CCl and TLR2 deficiency also reduced MyD88 expression

Finally, western blotting analysis were used to measure the protein expression level of MyD88 in the TNC in all tested three groups. The results showed that the expression level of MyD88 on the ipsilateral TNC of the surgery was significantly elevated in the dIoN-CCI surgery mice compared with that of sham mice (sham vs. dIoNCCI + vehicle, $\mathrm{t}_{6}=2.718, P=0.0347$ ), and upregulation of the expression of MyD88 was significantly decreased on day 5 after BoNT/A injection (day 19 after dIoN-CCI surgery) in mice (dIoN-CCI + vehicle vs. dIoN-CCI + BoNT $/ \mathrm{A}, \mathrm{t}_{6}=2.654, P=0.0378$; Fig. 9a). However, the expression of MyD88 did not change in the contralateral TNC of the surgery (sham vs. dIoN-CCI, $\mathrm{t}_{6}=0.9386$, $P=0.3842 ;$ dIoN-CCI vs. dIoN-CCI + BoNT/A, $\mathrm{t}_{6}=$ $0.5799, P=0.5831$; Fig. $9 b$ ). On day 14 after dIoN-CCI surgery in mice, the expression of MyD88 in the bilateral TNC of $\mathrm{Tlr}^{-/-}$mice was significantly reduced compared with that of wild-type mice (ipsilateral: $\mathrm{t}_{4}=2.923$, $P=0.0431$; Fig. 9c; contralateral: $t_{4}=3.438 ; P=0.0138$; 

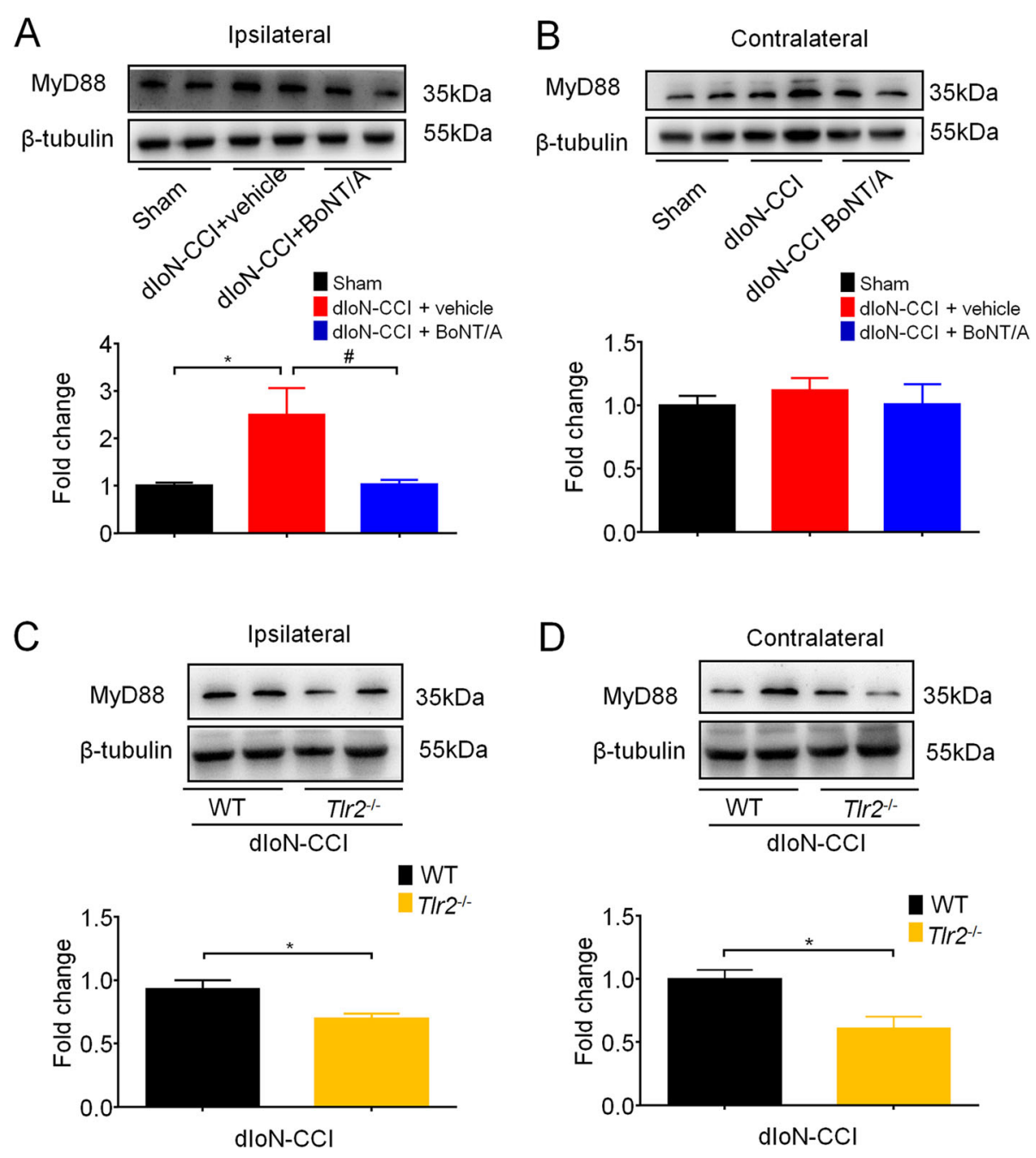

Fig. 9 Western blotting analysis showed the expression changes of Myd88 in the bilateral TNC in mice. a The expression of MyD88 was increased by dloN-CCl surgery compared with the sham group, and BoNT/A injection decreased this upregulation. $\mathbf{b}$ The expression of MyD88 did not change in the contralateral TNC by dloN-CCl surgery compared with the sham group. $\mathbf{c}-\mathbf{d}$ The expression of MyD88 in the ipsilateral and contralateral TNC was significantly reduced in T/r2 ${ }^{-1-}$ mice compared with wild-type mice following dloN-CCl surgery. Data was expressed as Mean \pm SEM. ( $n=4$ each group, ${ }^{*} P<0.05$ compared with the sham group. ${ }^{*} P<0.05$ compared with the TN group, unpaired Student's t-test) TNC, Trigeminal nucleus caudalis; dloN-CCl, Chronic constriction injury of distal infraorbital nerve; BoNT/A, Botulinum toxin A

Fig. 9d). Thus, these data indicated that MyD88 as downstream molecule of TLR signaling is involved in the pathogenesis of TN and BoNT/A injection could reduce the expression level of MyD88 in the ipsilateral TNC.

\section{Discussion}

In this study, we found that peripheral injury of the trigeminal nerve following dIoN-CCI surgery induced trigeminal neuropathic pain and anxiety-like behavior in mice, triggering microglia activation in the ipsilateral TNC, with the up-regulation of microglia TLR2 expression in the TNC. Importantly, peripheral s.c. injection of BoNT/A significantly attenuated bilateral mechanical pain hypersensitivity and anxiety-like behavior induced by dIoN-CCI surgery, which was associated with the inhibition of the microglia activation, decreased expression of TLR2, and reduced expression of several proinflammatory mediators in the TNC in mice.

The pathogenesis of primary TN are still largely unclear. To date, there are mainly three hypotheses to explain it. The first is the trigeminal nerve compression theory. The abnormal neurovascular compression of the trigeminal nerve at the pons stimulates the trigeminal nerve root, thus producing pain. Jannetta treated the patient of $\mathrm{TN}$ with microvascular decompression surgery, and pain disappeared after surgery, strongly supporting this theory [21]. The second is the central sensitization theory. The outbreak of TN have the characteristics of epileptic attack, and it is believed that pain may be 
associated with thalamic-cortico-trigeminal spinal tract nucleus irritative lesions [22]. The use of antiepileptic drugs in the treatment of TN may support this hypothesis [22]. The third is the neurodegeneration theory. The biopsy of the dysfunctional trigeminal nerve was found to produce demyelination changes and a "short-circuit" between two adjacent fibers of the demyelinated nerve may cause abnormal nerve transmission, leading to pain responding to a slight stimulus [23].

The previous clinical trials showed that BoNT/A was useful for the relief of TN in patients [24]. It was found that subcutaneous injections of BoNT/A produced analgesia lasting 60 days in 13 patients with $\mathrm{TN}$, who reduced or discontinued prophylactic medication and switched from multi-drug therapy to monotherapy without adverse effects [23]. Similarly, the previous study found that BoNT/A therapy was effective and safe in the treatment of elderly $(\geq 80$ years) TN patients at doses comparable to their more youthful counterparts (<60 years) [25]. Our present animal experiment confirmed that unilateral facial injection of BoNT/A attenuated bilateral mechanical pain hypersensitivity in a mouse TN model, which was induced by performing dIoN-CCI surgery in mice. Thus, our data indicated that local injection of BoNT/A may be effective for widespread pain in patients, including mirror image pain.

Epidemiological studies have shown that psychiatric disorders (such as anxiety) are common in patients with chronic pain [26-28]. Previous most studies on pain and anxiety have typically been conducted separately [29]. Chronic pain and anxiety are common comorbidities, and they could promote their development mutually [30, 31]. In the present study, we found that facial injection of BoNT/A could remarkable inhibit anxiety-like behavior in dIoN-CCI surgery mice. Our previous work showed that single facial injection of BoNT/A could have anti-depressant effects in depression model induced by chronic stress in mice [8]. Additionally, we also tested depression-like behavior in dIoN-CCI mouse model by using forced swimming test. However, we did not find significant difference in forced swimming test between sham and dIoN-CCI surgery mice. Nevertheless, BoNT/ A treatment attenuated both trigeminal neuropathic pain and accompanying anxiety-like behavior in mice, indicating BoNT/A treatment may be useful for the management of chronic pain-induced anxiety.

In the central nervous system, gliosis in response to injury often involves the proliferation or hypertrophy of glial cells [32]. Microgliosis exhibits profound morphological changes from branchial microglia to amoeboid microglia with enlarged cell bodies. In 1993, Erickson and his colleagues demonstrated significant microgliosis in the spinal cord and brainstem regions after nerve injury, by histochemical staining of OX-42 (antibodies recognizing CD11b) [33]. This is consistent with our findings on the morphological changes of microglia in the ipsilateral TNC induced by dIoN-CCI surgery in mice. The specific role of microglia in pain was discovered in 2003, when three independent studies showed the involvement of microglia in neuropathic pain after peripheral nerve injury [34-36]. There is growing evidence that microglia drive central sensitization through microglial mediators. Microglia are a major source of cytokines, including TNF- $\alpha$ and IL-1 $\beta$ [37]. The reduction or loss of inhibitory synaptic transmission in the pain circuitry of the spinal cord dorsal horn is also closely associated with central sensitization [38]. Notably, microglial mediators such as cytokines, PGE2 and BDNF, regulate inhibitory synaptic transmission in spinal cord dorsal horn neurons through presynaptic, postsynaptic and/or extrasynaptic mechanisms [37]. Proinflammatory cytokines strongly regulate inhibitory synaptic transmission in multiple sites in the dorsal horn of the spinal cord [39]. At the presynaptic level, IL-1 and IL-6 inhibit the frequency of spontaneous inhibitory postsynaptic currents (sIPSCs) in spinal pain circuits. At postsynaptic sites, IL-1 and IL-6 decreased the amplitude of sIPSCs. At extrasynaptic sites, GABA and glycine receptor activity could be inhibited by IL-1 $\beta$ [40, 41]. In addition, TNF- $\alpha$ rapidly inhibited the spontaneous action potentials of GABAergic neurons [42]. Zychowska et al. determine the effects of BoNT/A on pain-related behavior and the levels of glial markers and interleukins in the spinal cord and dorsal root ganglia (DRG) after chronic constriction injury (CCI) to the sciatic nerve in rats. It was found that injection of BoNT/A suppressed the CCI-induced upregulation of IL-18 and IL-1 $\beta$ in the spinal cord and/or DRG and increased the levels of IL10 and IL-1RA in the DRG. They suggest that BoNT/A significantly attenuates pain-related behavior and microglial activation and restores the neuroimmune balance by decreasing the levels of pro-nociceptive factors (IL-1 $\beta$ and IL-18) and increasing the levels of anti-nociceptive factors (IL-10 and IL-1RA) in the spinal cord and DRG [43]. In the present study, our RT-qPCR and immunostaining data demonstrated that BoNT/A treatment significantly inhibited microglia activation in the ipsilateral TNC induced by dIoN-CCI surgery in mice. In addition, our results showed that the mRNA expression of IL- $1 \beta$, TNF- $\alpha$ and IL- 6 in the ipsilateral TNC was also upregulated following dIoN-CCI surgery in mice, and BoNT/A treatment significantly inhibited the upregulation of these proinflammatory factors in the ipsilateral TNC. Thus, our findings indicated that peripheral administration of BoNT/A was able to suppress microgliamediated neuroinflammation in the central nerve system. 
BoNT/A therapy is a very useful strategy for the treatment of neuropathic pain, but how it induces functional recovery remains unknown. Neurohistology analysis confirmed that the distal part of the regenerative axon myelination had high expression of S100 after BoNT/A injection (S100 is used as a Schwann cell marker). Low doses of BoNT/A were not adequate to produce muscle dysfunction, instead, sensory-motor recovery was accelerated by stimulating the regeneration of axon myelination [44]. However, whether injection of BoNT/A promotes myelin regeneration or not warrants further investigation [45].

MyD88 is a key downstream adapter for most TLRs and IL-1Rs. Deletion of MyD88 in mice results in susceptibility to various pathogens [46]. Previous studies have shown that deletion of TLR4 can attenuate neuropathic pain in mice $[47,48]$. However, the role of TLR4 in the pathogenesis of TN was not investigated yet. After $\mathrm{TN}$, the deletion of TLR4 did not reduce the expression of MyD88, a downstream molecule of TLRs [49]. A recent study on TN showed that TLR8 activate ERK and $\mathrm{p} 38$, and further increase the expression of proinflammatory cytokines in the trigeminal ganglion neurons [50]. In our study, MyD88, which is an adaptor for all TLRs (except TLR3). dIoN-CCI surgery elevate the mRNA expression of TLR2 and TLR5 in the ipsilateral TNC in mice. After screening different TLRs expressed in the TNC, we focused our study on the role of TLR2 in the pathogenesis of TN [51], although there are no previous studies on the role of TLR2 in the pathogenesis of TN. Our study showed that TLR2 and MyD88 expression was upregulated in the TNC after dIoN-CCI surgery in mice. We established the same TN model on Tlr $2^{-/-}$ mice and wild-type mice to test mechanical pain and took the TNC tissue for western blotting. It was found that the bilateral mechanical pain hypersensitivity was significantly reduced and the upregulation of MyD88 expression was also decreased significantly in $T l r 2^{-/-}$mice. Interestingly, BoNT/A treatment was shown to reduce the mRNA and protein expression of TLR2 and its downstream MyD88 in the ipsilateral TNC in TN mouse model. Thereby, BoNT/A treatment attenuated trigeminal neuropathic pain, which may be associated the inhibition of TLR2mediated neuroinflammation in the TNC.

Finally, our findings suggest that unilateral ligation of branches of the trigeminal nerve can cause bilateral trigeminal nociceptive hyperalgesia in mice, which is also known as mirror image pain (Fig. 1). RT-qPCR assay also showed neuronal activation by elevated expression of c-Fos in the contralateral TNC following dIoN-CCI surgery. Injuries to peripheral nerves or spinal roots produce a number of breakdown products at the lesion and beyond, with a subsequent response from immunocompetent cells [52]. This inflammatory response releases a number of immunomodulatory cytokines that can be transported through the blood or cerebrospinal fluid (CSF) to contralateral sites in the body and affect the spinal roots or peripheral nerves [53, 54]. Neurons are elements of a highly organized neuronal network, and injury to peripheral neurons results in signals entering the central nervous system via neurons, which then eventually affect the contralateral ipsilateral neurons [55, 56]. Activation of glial cells is also thought to be involved in the phenomenon of ipsilateral or contralateral spread of pain [57]. The nerve-glial interaction is bidirectional. On the one hand, glial cells express different types of neurotransmitter receptors, which allow them to respond to neural signals [58]. On the other hand, glial cells produce many neuroactive mediators (e.g. proinflammatory cytokines and growth factors) [59]. Studies have shown that after peripheral injury, satellite glia in the contralateral dorsal root ganglion are activated by TNF- $\alpha$, which diffuses through the CSF from the injured side. Activated satellite glia produce additional nerve growth factor to enhance nociceptive excitability, inducing the pain of contralateral side [5]. We hypothesize that the relief of $\mathrm{TN}$ on the contralateral side of surgery after BoNT/A injection is due to the reduction of TNF- $\alpha$ and other inflammatory factors released from the TNC site on the side of surgery. Then the diffusion of inflammatory factors through the cerebrospinal fluid is not sufficient to activate the contralateral satellite glia to release NGF, so the contralateral central neurons remain stationary. This is what we need to prove in the future.

Of note, there are several limitations of our study. First, we only investigated the therapeutic mechanism of BoNT/A for trigeminal neuralgia in male mice. Given more and more studies employed both male and female animals, it remains to be investigated whether BoNT/A exerts similar therapeutic effects in female animals. Whether inhibition of microglia contributes to the therapeutic effects of BoNT/A in both male and female animal warrants further investigation. Second, there are several other TN animal models besides dIoN-CCI surgery developed in mice. Therapeutic effects of BoNT/A should be confirmed by using different $\mathrm{TN}$ animal models. Third, it was noticed after injection of BoNT/A, flaccid paralysis of the facial muscles at the injection site was observed. The side effects of BoNT/A therapy for TN should be investigated in the future. Finally, the direct inhibitory effects of BoNT/A on microglia and the underlying mechanisms in vitro should be explored.

\section{Conclusion}

In conclusion, our study showed that TN caused the activation of microglia, upregulation of TLR2 and MyD88 expression, causing elevated expression of inflammatory factors in the ipsilateral TNC site following dIoN-CCI 
surgery in mice. Peripheral unilateral injection of BoNT/ A could inhibit bilateral trigeminal neuropathic pain and anxiety-like behaviors, which is associated with the inhibition of microglia activation, the upregulation of expression of TLR2, its downstream of MyD88 and proinflammatory factors in the ipsilateral TNC and inhibit the activation of neurons in the contralateral TNC following dIoN-CCI surgery in mice. Therefore, we have provided preclinical evidence for the effectiveness of BoNT/A therapy on the management of $\mathrm{TN}$ and its comorbidity anxiety.

\begin{abstract}
Abbreviations
TN: Trigeminal neuralgia; BoNT/A: Botulinum toxin type A; MAPK: Mitogenactivated protein kinases; TLRs: Toll-like receptors; TLR2: Toll-like receptor 2; Myd88: Myeloid differentiation factor; GFAP: Glial fibrillary acidic protein; IBA1: Ion calcium-binding bridging molecule 1; TNF-a: Tumour Necrosis Factor alpha; IL-1ß: Interleukin 1 beta; IL-6: Interleukin-6; CCI: Chronic constriction injury; loN: Infraorbital nerve; TG: Trigeminal ganglion; TNC: Trigeminal nucleus caudalis
\end{abstract}

\section{Acknowledgements}

None.

\section{Authors' contributions}

WJC, JQN, YTC, WJD, YYX, JL, WFL and TL contributed to the work design, performed experiments, and analyzed and interpreted data from all the experiments. Animal behavior experiments were performed by WJC, JQN, YTC. Molecular biology experiments were performed by WJC, JQN, YTC, WJD. WJC, WFL and TL wrote and completed the manuscript. All authors critically revised and approved the final manuscript and agreed to take responsibility for all aspects of the work.

\section{Funding}

This work was supported by Suzhou science and technology plan key technology application research project (SS2019060), and the National Natural Science Foundation of China (81671270; 81870874).

\section{Availability of data and materials}

The datasets used and/or analyzed during the current study are available from the corresponding author on reasonable request.

\section{Declarations}

\section{Ethics approval and consent to participate}

The animal study was reviewed and approved by the Ethics Committee for the Use of Experimental Animals in Soochow University Animal Committee.

\section{Consent for publication}

Not applicable.

\section{Competing interests}

The authors declare that they have no competing interests.

\section{Author details}

${ }^{1}$ Department of Neurology, The Second Affiliated Hospital of Soochow University, 1055 Sanxiang Road, Suzhou 215004, China. ${ }^{2}$ The First Affiliated Hospital of USTC, Division of Life Sciences and Medicine, University of Science and Technology of China, Hefei 230001, Anhui, China. ${ }^{3}$ Changzhou Hygiene Vocational Technology College, Changzhou 213002, China. ${ }^{4}$ Jiangsu Key Laboratory of Translational Research and Therapy for

Neuro-Psychiatric-Diseases, Soochow University, Suzhou 215021, China. ${ }^{5}$ Institute of Pain Medicine and Special Environmental Medicine, Nantong University, Nantong 226019, China. ${ }^{6}$ College of Life Sciences, Yanan University, Yanan 716000, China. ${ }^{7}$ Suzhou Key Laboratory of Intelligent Medicine and Equipment, Suzhou 215123, China.
Received: 25 February 2021 Accepted: 6 May 2021

Published online: 17 May 2021

\section{References}

1. Burmeister J, Holle D, Bock E, Ose C, Diener H-C, Obermann M (2015) Botulinum neurotoxin type $A$ in the treatment of classical trigeminal neuralgia (BoTN): study protocol for a randomized controlled trial. Trials 16(1):550. https://doi.org/10.1186/s13063-015-1052-z

2. Jorns TP, Zakrzewska JM (2007) Evidence-based approach to the medical management of trigeminal neuralgia. Br J Neurosurg 21(3):253-261. https:// doi.org/10.1080/02688690701219175

3. Bond AE, Zada G, Gonzalez AA, Hansen C, Giannotta SL (2010) Operative strategies for minimizing hearing loss and other major complications associated with microvascular decompression for trigeminal neuralgia. World Neurosurg 74(1):172-177. https://doi.org/10.1016/j.wneu.2010.05.001

4. Ishikawa T, Eto K, Kim SK, Wake H, Takeda I, Horiuchi H, Moorhouse AJ, Ishibashi H, Nabekura J (2018) Cortical astrocytes prime the induction of spine plasticity and mirror image pain. Pain 159(8):1592-1606. https://doi. org/10.1097/j.pain.0000000000001248

5. Cheng $C-F$, Cheng J-K, Chen C-Y, Lien C-C, Chu D, Wang S-Y, Tsaur ML (2014) Mirror-image pain is mediated by nerve growth factor produced from tumor necrosis factor alpha-activated satellite glia after peripheral nerve injury. Pain 155(5):906-920. https://doi.org/10.1016/j.pain.2014.01.010

6. Wu C-j, Shen J-h, Chen Y, Y-j L (2011) Comparison of two different formulations of botulinum toxin a for the treatment of blepharospasm and hemifacial spasm. Turk Neurosurg 21(4):625-629. https://doi.org/10.5137/101 9-5149.JTN.4524-11.1

7. Aoki KR (2003) Evidence for antinociceptive activity of botulinum toxin type A in pain management. Headache 43:S9-S15

8. Li Y, Liu J, Liu X, Su C-J, Zhang Q-L, Wang Z-H, Cao LF, Guo XY, Huang Y, Luo W, Liu T (2019) Antidepressant-like action of single facial injection of botulinum neurotoxin a is associated with augmented 5-HT levels and BDNF/ERK/CREB pathways in mouse brain. Neurosci Bull 35(4):661-672. https://doi.org/10.1007/s12264-019-00367-8

9. Sheng H-Y, Lv S-S, Cai Y-Q, Shi W, Lin W, Liu T-T, Lv N, Cao H, Zhang L, Zhang YQ (2020) Activation of ventrolateral orbital cortex improves mouse neuropathic pain-induced anxiodepression. JCI Insight 5(19):e133625. https://doi.org/10.1172/jci.insight.133625

10. Ji RR, Nackley A, Huh Y, Terrando N, Maixner W (2018) Neuroinflammation and central sensitization in chronic and widespread pain. Anesthesiology 129(2):343-366. https://doi.org/10.1097/ALN.0000000000002130

11. Chen G, Zhang YQ, Qadri YJ, Serhan CN, Ji RR (2018) Microglia in pain: detrimental and protective roles in pathogenesis and resolution of pain. Neuron 100(6):1292-1311. https://doi.org/10.1016/j.neuron.2018.11.009

12. Akira S (2013) Pathogen recognition receptors and innate immunity. Xenotransplantation 20:351-351

13. Hayashi F, Smith KD, Ozinsky A, Hawn TR, Yi EC, Goodlett DR, Eng JK, Akira S, Underhill DM, Aderem A (2001) The innate immune response to bacterial flagellin is mediated by toll-like receptor 5. Nature 410(6832):1099-1103. https://doi.org/10.1038/35074106

14. Naert G, Laflamme N, Rivest S (2009) Toll-like receptor 2-independent and MyD88-dependent the mouse brain. J Innate Immun 1(5):480-493. https:// doi.org/10.1159/000225990

15. Kim YJ, Kim J-H, Lee K-J, Choi M-M, Kim YH, Rhie G-E, Yoo CK, Cha K, Shin NR (2015) Botulinum neurotoxin type A induces TLR2-mediated inflammatory responses in macrophages. PLoS One 10(4):e0120840. https:// doi.org/10.1371/journal.pone.0120840

16. Ding W, You Z, Shen S, Yang J, Lim G, Doheny JT, Chen L, Zhu S, Mao J (2017) An improved rodent model of trigeminal neuropathic pain by unilateral chronic constriction injury of distal infraorbital nerve. J Pain 18(8): 899-907. https://doi.org/10.1016/j.jpain.2017.02.427

17. Kilkenny C, Browne W, Cuthill IC, Emerson M, Altman DG (2010) Animal research: reporting in vivo experiments: the ARRIVE guidelines. $\mathrm{Br} J$ Pharmacol 160:1577-1579. https://doi.org/10.1111/j.1476-5381.2010.00872.x

18. Kraeuter A-K, Guest PC, Sarnyai Z (2019) The elevated plus maze test for measuring anxiety-like behavior in rodents. Methods Mol Biol 1916:69-74

19. Xu ZZ, Kim YH, Bang SS, Zhang Y, Berta T, Wang F, Oh SB, Ji RR (2015) Inhibition of mechanical allodynia in neuropathic pain by TLR5-mediated Afiber blockade. Nat Med 21(11):1326-1331. https://doi.org/10.1038/nm.3978

20. Liu T, Han QJ, Chen G, Huang Y, Zhao LX, Berta T, Gao YJ, Ji RR (2016) Tolllike receptor 4 contributes to chronic itch, alloknesis, and spinal astrocyte 
activation in male mice. Pain 157(4):806-817. https://doi.org/10.1097/j.pain 0000000000000439

21. Jannetta PJ (2007) Arterial compression of the trigeminal nerve at the pons in patients with trigeminal neuralgia. J Neurosurg 107(1):216-219. https:// doi.org/10.3171/JNS-07/07/0216

22. Idanpaan-Heikkila JJ, Guilbaud G (1999) Pharmacological studies on a rat model of trigeminal neuropathic pain: baclofen, but not carbamazepine, morphine or tricyclic antidepressants, attenuates the allodynia-like behaviour. Pain 79(2):281-290. https://doi.org/10.1016/S0304-3959(98)001729

23. Devor M, Govrin-Lippmann R, Rappaport ZH (2002) Mechanism of trigeminal neuralgia: an ultra-structural analysis, of trigeminal root specimens obtained during microvascular decompression surgery. J Neurosurg 96(3):532-543. https://doi.org/10.3171/jns.2002.96.3.0532

24. Piovesan EJ, Teive HG, Kowacs PA, Della Coletta MV, Werneck LC, Silberstein SD (2005) An open study of botulinum - a toxin treatment of trigeminal neuralgia. Neurology 65(8):1306-1308. https://doi.org/10.1212/01.wnl.00001 80940.98815 .74

25. Liu J, Xu Y-Y, Zhang Q-L, Luo W-F (2018) Efficacy and safety of botulinum toxin type a in treating patients of advanced age with idiopathic trigeminal neuralgia. Pain Res Manag 2018:7365148

26. Price DD (2000) Neuroscience - psychological and neural mechanisms of the affective dimension of pain. Science 288(5472):1769-1772. https://doi. org/10.1126/science.288.5472.1769

27. Bushnell MC, Ceko M, Low LA (2013) Cognitive and emotional control of pain and its disruption in chronic pain. Nat Rev Neurosci 14(7):502-511. https://doi.org/10.1038/nrn3516

28. Velly AM, Mohit S (2018) Epidemiology of pain and relation to psychiatric disorders. Prog Neuro-Psychopharmacol Biol Psychiatry 87(Pt B):159-167. https://doi.org/10.1016/j.pnpbp.2017.05.012

29. Zhuo M (2016) Neural mechanisms underlying anxiety-chronic pain interactions. Trends Neurosci 39(3):136-145. https://doi.org/10.1016/j.tins.201 6.01 .006

30. Bair MJ, Wu JW, Damush TM, Sutherland JM, Kroenke K (2008) Association of depression and anxiety alone and in combination with chronic musculoskeletal pain in primary care patients. Psychosom Med 70(8):890897. https://doi.org/10.1097/PSY.0b013e318185c510

31. Bruffaerts $R$, Demyttenaere $K$, Kessler RC, Tachimori H, Bunting B, Hu C, Florescu S, Haro JM, Lim CCW, Kovess-Masfety V, Levinson D, Medina Mora ME, Piazza M, Piotrowski P, Posada-Villa J, Salih Khalaf M, ten Have M, Xavier M, Scott KM (2015) The associations between preexisting mental disorders and subsequent onset of chronic headaches: a worldwide epidemiologic perspective. J Pain 16(1):42-52. https://doi.org/10.1016/j.jpain.2014.10.002

32. Bellver-Landete $V$, Bretheau F, Mailhot $B$, Vallieres $N$, Lessard M, Janelle ME et al (2019) Microglia are an essential component of the neuroprotective scar that forms after spinal cord injury. Nat Commun 10:18

33. Eriksson NP, Persson JKE, Svensson M, Arvidsson J, Molander C, Aldskogius $H$ (1993) A quantitative-analysis of the microglial cell reaction in central primary sensory projection territories following peripheral-nerve injury in the adult-rat. Exp Brain Res 96(1):19-27. https://doi.org/10.1007/BF00230435

34. Tsuda M, Shigemoto-Mogami Y, Koizumi S, Mizokoshi A, Kohsaka S, Salter MW, Inoue K (2003) P2X(4) receptors induced in spinal microglia gate tactile allodynia after nerve injury. Nature 424(6950):778-783. https://doi.org/10.103 8/nature01786

35. Jin SX, Zhuang ZY, Woolf CJ, Ji RR (2003) P38 mitogen-activated protein kinase is activated after a spinal nerve ligation in spinal cord microglia and dorsal root ganglion neurons and contributes to the generation of neuropathic pain. J Neurosci 23(10):4017-4022. https://doi.org/10.1523/ JNEUROSCI.23-10-04017.2003

36. Raghavendra V, Tanga F, DeLeo JA (2003) Inhibition of microglial activation attenuates the development but not existing hypersensitivity in a rat model of neuropathy. J Pharmacol Exp Ther 306(2):624-630. https://doi.org/1 0.1124 /jpet.103.052407

37. Hanisch UK (2002) Microglia as a source and target of cytokines. Glia 40(2): 140-155. https://doi.org/10.1002/glia.10161

38. Latremoliere A, Woolf CJ (2009) Central sensitization: a generator of pain hypersensitivity by central neural plasticity. J Pain 10(9):895-926. https://doi. org/10.1016/j.jpain.2009.06.012

39. Ji RR, Berta T, Nedergaard M (2013) Glia and pain: is chronic pain a gliopathy? Pain 154(Supplement 1):S10-S28. https://doi.org/10.1016/j.pain.2 013.06 .022
40. Kawasaki Y, Zhang L, Cheng JK, Ji RR (2008) Cytokine mechanisms of central sensitization: distinct and overlapping role of interleukin-1 beta, interleukin6 , and tumor necrosis factor-beta in regulating synaptic and neuronal activity in the superficial spinal cord. J Neurosci 28(20):5189-5194. https:// doi.org/10.1523/JNEUROSCI.3338-07.2008

41. Yan $X$, Jiang $E$, Weng $H-R$ (2015) Activation of toll like receptor 4 attenuates GABA synthesis and postsynaptic GABA receptor activities in the spinal dorsal horn via releasing interleukin-1 beta. J Neuroinflammation 12(1):222. https://doi.org/10.1186/s12974-014-0222-3

42. Zhang H, Nie H, Dougherty PM (2011) A p38 mitogen-activated protein kinase-dependent mechanism of disinhibition in spinal synaptic transmission induced by tumor necrosis factor-alpha. J Neurosci 30:1284412855

43. Zychowska M, Rojewska E, Makuch W, Luvisetto S, Pavone F, Marinelli S, Przewlocka B, Mika J (2016) Participation of pro- and anti-nociceptive interleukins in botulinum toxin A-induced analgesia in a rat model of neuropathic pain. Eur J Pharmacol 791:377-388. https://doi.org/10.1016/j. ejphar.2016.09.019

44. Cobianchi S, Jaramillo J, Luvisetto S, Pavone F, Navarro X (2017) Botulinum neurotoxin A promotes functional recovery after peripheral nerve injury by increasing regeneration of myelinated fibers. Neuroscience 359:82-91. https://doi.org/10.1016/j.neuroscience.2017.07.011

45. Dong F, Liu D, Jiang F, Liu Y, Wu X, Qu X, Liu J, Chen Y, Fan H, Yao R (2021) Conditional deletion of foxg1 alleviates demyelination and facilitates remyelination via the Wnt signaling pathway in cuprizone-induced demyelinated mice. Neurosci Bull 37(1):15-30. https://doi.org/10.1007/s122 64-020-00583-7

46. von Bernuth H, Picard C, Jin ZB, Pankla R, Xiao H, Ku CL, Chrabieh M, Mustapha IB, Ghandil P, Camcioglu Y, Vasconcelos J, Sirvent N, Guedes M, Vitor AB, Herrero-Mata MJ, Arostegui Jl, Rodrigo C, Alsina L, Ruiz-Ortiz E, Juan M, Fortuny C, Yague J, Anton J, Pascal M, Chang HH, Janniere L, Rose Y, Garty BZ, Chapel H, Issekutz A, Marodi L, Rodriguez-Gallego C, Banchereau J, Abel L, Li X, Chaussabel D, Puel A, Casanova JL (2008) Pyogenic bacterial infections in humans with MyD88 deficiency. Science 321(5889):691-696. https://doi.org/10.1126/science.1158298

47. Bruno K, Woller SA, Miller Yl, Yaksh TL, Wallace M, Beaton G, Chakravarthy K (2018) Targeting toll-like receptor-4 (TLR4)-an emerging therapeutic target for persistent pain states. Pain 159(10):1908-1915. https://doi.org/10.1097/j. pain.0000000000001306

48. Jia GL, Huang Q, Cao YN, Xie CS, Shen YJ, Chen JL, Lu JH, Zhang MB, Li J, Tao YX, Cao H (2020) Cav-1 participates in the development of diabetic neuropathy pain through the TLR4 signaling pathway. J Cell Physiol 235(3): 2060-2070. https://doi.org/10.1002/jcp.29106

49. Hu TT, Wang RR, Tang YY, Wu YX, Yu J, Hou WW, Lou GD, Zhou YD, Zhang SH, Chen Z (2018) TLR4 deficiency abrogated widespread tactile allodynia, but not widespread thermal hyperalgesia and trigeminal neuropathic pain after partial infraorbital nerve transection. Pain 159(2):273-283. https://doi. org/10.1097/j.pain.0000000000001100

50. Zhao L-X, Jiang M, Bai X-Q, Cao D-L, Wu X-B, Zhang J, Guo JS, Chen TT, Wang J, Wu H, Gao YJ, Zhang ZJ (2021) TLR8 in the trigeminal ganglion contributes to the maintenance of trigeminal neuropathic pain in mice. Neurosci Bull 37(4):550-562. https://doi.org/10.1007/s12264-020-00621-4

51. Moreau N, Dieb W, Mauborgne A, Bourgoin S, Villanueva L, Pohl M, Boucher Y (2017) Hedgehog pathway-mediated vascular alterations following trigeminal nerve injury. J Dent Res 96(4):450-457. https://doi.org/10.1177/ 0022034516679395

52. Bombeiro AL, Santini JC, Thome R, Ferreira ERL, Nunes SLO, Moreira BM et al (2016) Enhanced immune response in immunodeficient mice improves peripheral nerve regeneration following axotomy. Front Cell Neurosci 10:S1-S14

53. Skouen JS, Brisby H, Otani K, Olmarker K, Rosengren L, Rydevik B (1999) Protein markers in cerebrospinal fluid in experimental nerve root injury - a study of slow-onset chronic compression effects or the biochemical effects of nucleus pulposus on sacral nerve roots. Spine 24(21):2195-2200. https:// doi.org/10.1097/00007632-199911010-00004

54. Cornefjord M, Nyberg F, Rosengren L, Brisby H (2004) Cerebrospinal fluid biomarkers in experimental spinal nerve root injury. Spine 29(17):1862-1868. https://doi.org/10.1097/01.brs.0000137070.16592.b2

55. Donaldson LF, McQueen DS, Seckl JR (1995) Neuropeptide gene-expression and capsaicin-sensitive primary afferents - maintenance and spread of adjuvant arthritis in the rat. J Physiol Lond 486(2):473-482. https://doi.org/1 0.1113/jphysiol.1995.sp020826 
56. Kelly S, Dunham JP, Donaldson LF (2007) Sensory nerves have altered function contralateral to a monoarthritis and may contribute to the symmetrical spread of inflammation. Eur J Neurosci 26(4):935-942. https:// doi.org/10.1111/j.1460-9568.2007.05737.x

57. Hansson E (2006) Could chronic pain and spread of pain sensation be induced and maintained by glial activation? Acta Physiol 187(1-2):321-327. https://doi.org/10.1111/j.1748-1716.2006.01568.x

58. Kettenmann H, Hanisch UK, Noda M, Verkhratsky A (2011) Physiology of microglia. Physiol Rev 91(2):461-553. https://doi.org/10.1152/physrev.00011.2010

59. Hashizume H, DeLeo JA, Colburn RW, Weinstein JN (2000) Spinal glial activation and cytokine expression after lumbar root injury in the rat. Spine 25(10):1206-1217. https://doi.org/10.1097/00007632-200005150-00003

\section{Publisher's Note}

Springer Nature remains neutral with regard to jurisdictional claims in published maps and institutional affiliations.

- fast, convenient online submission

- thorough peer review by experienced researchers in your field

- rapid publication on acceptance

- support for research data, including large and complex data types

- gold Open Access which fosters wider collaboration and increased citations

- maximum visibility for your research: over $100 \mathrm{M}$ website views per year

At BMC, research is always in progress.

Learn more biomedcentral.com/submissions 